Review

\title{
On the Use of Quantum Chemistry for the Determination of Propagation, Copolymerization, and Secondary Reaction Kinetics in Free Radical Polymerization
}

\author{
Evangelos Mavroudakis ${ }^{1,2}$, Danilo Cuccato ${ }^{3, *}$ and Davide Moscatelli ${ }^{1}$ \\ ${ }^{1}$ Dipartimento di Chimica, Materiali e Ingegneria Chimica "Giulio Natta”, Politecnico di Milano, \\ Milano 20131, Italy; E-Mails: evangelos.mavroudakis@polimi.it (E.M.); \\ davide.moscatelli@polimi.it (D.M.) \\ 2 Environmental Research Laboratory, National Center for Scientific Research "Demokritos", \\ Agia Paraskevi Attikis 15310, Greece \\ ${ }^{3}$ Institute for Chemical and Bioengineering, Department of Chemistry and Applied Biosciences, \\ Eidgenössische Technische Hochschule Zurich, Zurich 8093, Switzerland \\ * Author to whom correspondence should be addressed; E-Mail: danilo.cuccato@chem.ethz.ch; \\ Tel.: +41-446-326-183; Fax: +41-446-321-082.
}

Academic Editor: Graeme Moad

Received: 3 June 2015 / Accepted: 6 September 2015 / Published: 17 September 2015

\begin{abstract}
Throughout the last 25 years, computational chemistry based on quantum mechanics has been applied to the investigation of reaction kinetics in free radical polymerization (FRP) with growing interest. Nowadays, quantum chemistry (QC) can be considered a powerful and cost-effective tool for the kinetic characterization of many individual reactions in FRP, especially those that cannot yet be fully analyzed through experiments. The recent focus on copolymers and systems where secondary reactions play a major role has emphasized this feature due to the increased complexity of these kinetic schemes. QC calculations are well-suited to support and guide the experimental investigation of FRP kinetics as well as to deepen the understanding of polymerization mechanisms. This paper is intended to provide an overview of the most relevant QC results obtained so far from the investigation of FRP. A comparison between computational results and experimental data is given, whenever possible, to emphasize the performances of the two approaches in the prediction of kinetic data. This work provides a comprehensive database of reaction rate parameters of FRP to assist in the development of advanced models of polymerization and experimental studies on the topic.
\end{abstract}


Keywords: free radical polymerization; quantum chemistry; reaction kinetics; copolymerization; secondary reactions; pulsed-laser polymerization

\section{Introduction}

One of the most common and versatile polymerization processes for the production of macromolecular materials is the free radical polymerization (FRP). High molecular weight polymers can be easily produced even under mild polymerization conditions using only monomer and a radical initiator. Since the 1980s, half of the production of synthetic polymers has been carried out through FRP processes [1]. Nowadays, still $45 \%$ of the manufactured plastic materials and $40 \%$ of synthetic rubber are obtained by FRP processes [2]. Such processes can exploit the large variety of vinyl compounds available on the market.

On the other hand, the complexity of the kinetic scheme of FRP and the large number of reactions involved often make it difficult to achieve a nice regulation of molecular weight distribution and polymer morphology. Basically, FRP is a network of reaction steps that involve a large number of different species, including very reactive radicals, which may vary in nature within the same system. An indefinite number of reaction pathways is therefore possible. The knowledge of the corresponding kinetics is very valuable for the prediction of certain final polymer features, from the chain microstructure to the macroscopic properties [3].

The accurate determination of these kinetic parameters is as essential for the synthesis of polymer materials with specific properties as it is complicated. The need for an experimental procedure that provided an accurate estimation of the individual rate coefficients of the reactions that govern the FRP encouraged the development of the pulsed laser polymerization (PLP) technique [4]. Since its invention in the late 1980s, PLP-based techniques have substantially improved the quality of the experimental measurements of rate parameters in FRP, particularly for propagation reactions. These techniques have also been widely used for the study of the homopolymerization and copolymerization of various monomers [5-18].

Nowadays, PLP coupled with size exclusion chromatography (SEC) is the method recommended by the International Union of Pure and Applied Chemistry (IUPAC) for the measurements of propagation rate coefficients [19-22]. The propagation rate coefficients for various important monomers evaluated by PLP are listed in Table 1. It should be noted that the propagation kinetics of water-soluble monomers can be largely affected by their concentration in aqueous medium. Therefore, the monomer concentration of such systems is also indicated in Table 1. Additionally, PLP coupled with other analytical methods allows the investigation of a relatively large number of reactions in FRP. In this regard, it is important to note the impact of the use of PLP coupled with electron paramagnetic resonance (EPR) on the understanding and kinetic characterization of termination and transfer reaction steps, particularly those involving mid-chain radicals (MCR) [12,14-17]. 
Table 1. Propagation rate coefficients, $k$, and Arrhenius parameters (activation energy, $E_{\mathrm{a}}$, and pre-exponential factor, $A$ ) of the free radical polymerization of various monomers estimated through pulsed laser polymerization (PLP) experiments.

\begin{tabular}{|c|c|c|c|c|c|c|}
\hline Monomer & Year & $\begin{array}{c}E_{\mathrm{a}} \\
(\mathrm{kJ} / \mathrm{mol})\end{array}$ & $\begin{array}{l}\lg (A)(A, \\
\mathrm{L} / \mathrm{mol} / \mathrm{s})\end{array}$ & $\begin{array}{c}k\left(50{ }^{\circ} \mathrm{C}\right. \\
\mathrm{L} / \mathrm{mol} / \mathrm{s})\end{array}$ & $\begin{array}{c}\text { Temperature } \\
\text { range }\end{array}$ & Reference \\
\hline MA & 2013 & 17.3 & 7.15 & $2.25 \times 10^{4}$ & -28 to $60^{\circ} \mathrm{C}$ & [18] \\
\hline BA & 2008 & 18.1 & 7.36 & $2.71 \times 10^{4}$ & 10 to $70^{\circ} \mathrm{C}$ & [7] \\
\hline EHA & 2010 & 15.8 & 6.96 & $2.54 \times 10^{4}$ & 10 to $82^{\circ} \mathrm{C}$ & [23] \\
\hline HEA & 2012 & 16.8 & 7.24 & $3.33 \times 10^{4}$ & 20 to $60^{\circ} \mathrm{C}$ & {$[24]$} \\
\hline MMA & 1997 & 22.4 & 6.43 & $6.42 \times 10^{2}$ & -1 to $90^{\circ} \mathrm{C}$ & [21] \\
\hline EMA & 2000 & 23.4 & 6.61 & $6.69 \times 10^{2}$ & 6 to $90{ }^{\circ} \mathrm{C}$ & [22] \\
\hline BMA & 2000 & 22.9 & 6.58 & $7.53 \times 10^{2}$ & -20 to $90{ }^{\circ} \mathrm{C}$ & [22] \\
\hline DMA & 2000 & 21.0 & 6.40 & $1.01 \times 10^{3}$ & 9 to $90^{\circ} \mathrm{C}$ & [22] \\
\hline HEMA & 1998 & 21.9 & 6.95 & $2.56 \times 10^{3}$ & -4 to $70^{\circ} \mathrm{C}$ & [25] \\
\hline iBMA & 1997 & 22.5 & 6.50 & $7.26 \times 10^{2}$ & 10 to $90^{\circ} \mathrm{C}$ & [26] \\
\hline iDMA & 1997 & 20.8 & 6.34 & $9.47 \times 10^{2}$ & 10 to $90{ }^{\circ} \mathrm{C}$ & [26] \\
\hline EHMA & 1997 & 20.4 & 6.27 & $9.35 \times 10^{2}$ & 10 to $90{ }^{\circ} \mathrm{C}$ & [26] \\
\hline EEMA & 2010 & 24.1 & 6.73 & $6.80 \times 10^{2}$ & 4 to $61{ }^{\circ} \mathrm{C}$ & [27] \\
\hline PEGEEMA & 2010 & 24.4 & 6.97 & $1.06 \times 10^{3}$ & 4 to $61{ }^{\circ} \mathrm{C}$ & [27] \\
\hline CHMA & 2003 & 23.0 & 6.80 & $1.20 \times 10^{3}$ & 10 to $91^{\circ} \mathrm{C}$ & [28] \\
\hline GMA & 2003 & 22.9 & 6.79 & $1.22 \times 10^{3}$ & 20 to $90^{\circ} \mathrm{C}$ & [28] \\
\hline BzMA & 2003 & 22.9 & 6.83 & $1.34 \times 10^{3}$ & 6 to $90^{\circ} \mathrm{C}$ & [28] \\
\hline iBoMA & 2003 & 23.1 & 6.79 & $1.13 \times 10^{3}$ & 0 to $90^{\circ} \mathrm{C}$ & [28] \\
\hline $\mathrm{AA}\left(10 \% w / w, \mathrm{H}_{2} \mathrm{O}\right)$ & 2003 & 15.4 & 7.82 & $2.14 \times 10^{5}$ & 2 to $20^{\circ} \mathrm{C}$ & [29] \\
\hline $\operatorname{NVP}\left(40 \% w / w, \mathrm{H}_{2} \mathrm{O}\right)$ & 2014 & 18.4 & 6.97 & $9.87 \times 10^{3}$ & -5 to $80^{\circ} \mathrm{C}$ & {$[30,31]$} \\
\hline $\operatorname{NVF}\left(40 \% w / w, \mathrm{H}_{2} \mathrm{O}\right)$ & 2010 & 20.2 & 6.79 & $3.34 \times 10^{3}$ & 5 to $60^{\circ} \mathrm{C}$ & [32] \\
\hline AAm & 2005 & 13.4 & 7.20 & $1.08 \times 10^{5}$ & 6 to $50{ }^{\circ} \mathrm{C}$ & [33] \\
\hline TRIS & 2000 & 19.9 & 6.16 & $8.74 \times 10^{2}$ & 15 to $50^{\circ} \mathrm{C}$ & [34] \\
\hline NVC & 2003 & 25.3 & 8.00 & $8.10 \times 10^{3}$ & 0 to $50{ }^{\circ} \mathrm{C}$ & [35] \\
\hline VneoD & 1997 & 22.2 & 7.31 & $5.25 \times 10^{3}$ & -21 to $21^{\circ} \mathrm{C}$ & [36] \\
\hline VAc & 1995 & 20.7 & 7.17 & $6.64 \times 10^{3}$ & 10 to $60^{\circ} \mathrm{C}$ & [37] \\
\hline 1,3-butadiene & 1989 & 35.7 & 7.90 & $1.34 \times 10^{2}$ & 30 to $60^{\circ} \mathrm{C}$ & [38] \\
\hline MAN & 1995 & 29.7 & 6.43 & $4.23 \times 10^{1}$ & 10 to $60^{\circ} \mathrm{C}$ & [39] \\
\hline MAA & 1997 & 17.7 & 5.78 & $8.27 \times 10^{2}$ & 20 to $60^{\circ} \mathrm{C}$ & [40] \\
\hline AN & 2010 & 15.4 & 6.25 & $5.75 \times 10^{3}$ & 2 to $76^{\circ} \mathrm{C}$ & [41] \\
\hline St & 1995 & 32.5 & 7.63 & $2.37 \times 10^{2}$ & -12 to $93{ }^{\circ} \mathrm{C}$ & {$[42]$} \\
\hline
\end{tabular}


Table 1. Cont.

\begin{tabular}{ccccccc}
\hline Monomer & Year & $\begin{array}{c}\boldsymbol{E}_{\mathbf{a}} \\
(\mathbf{k J} / \mathbf{m o l})\end{array}$ & $\begin{array}{c}\mathbf{l g}(\boldsymbol{A})(\boldsymbol{A}, \\
\mathbf{L} / \mathbf{m o l} / \mathbf{s})\end{array}$ & $\begin{array}{c}\boldsymbol{k}\left(\mathbf{5 0}{ }^{\circ} \mathbf{C},\right. \\
\mathbf{L} / \mathbf{m o l} / \mathbf{s})\end{array}$ & $\begin{array}{c}\text { Temperature } \\
\text { range }\end{array}$ & Reference \\
\hline 4-MeO-St & 1999 & 34.9 & 7.80 & $1.43 \times 10^{2}$ & 20 to $40^{\circ} \mathrm{C}$ & {$[43]$} \\
4-Me-St & 1999 & 32.4 & 7.45 & $1.62 \times 10^{2}$ & 20 to $40{ }^{\circ} \mathrm{C}$ & {$[43]$} \\
4-F-St & 1999 & 32.0 & 7.54 & $2.32 \times 10^{2}$ & 20 to $40{ }^{\circ} \mathrm{C}$ & {$[43]$} \\
4-Cl-St & 1999 & 32.1 & 7.65 & $2.88 \times 10^{2}$ & 20 to $40^{\circ} \mathrm{C}$ & {$[43]$} \\
4-Br-St & 1999 & 33.9 & 7.98 & $3.14 \times 10^{2}$ & 20 to $40^{\circ} \mathrm{C}$ & {$[43]$} \\
Chloroprene & 1993 & 26.6 & 7.29 & $9.73 \times 10^{2}$ & 10 to $55^{\circ} \mathrm{C}$ & {$[44]$} \\
\hline
\end{tabular}

St, styrene; MA, methyl acrylate; BA, butyl acrylate; MMA, methy methacrylate; EMA, ethyl methacrylate; BMA, butyl methacrylate; DMA, dodecyl methacrylate; EHA, ethylhexyl acrylate; HEA, hydroxyethyl acrylate; HEMA, 2-hydroxyethyl methacrylate; AA, acrylic acid; iBMA, isobutyl methacrylate; iDMA, isodecyl methacrylate; EHMA, 2-ethylhexyl methacrylate; AAm, acrylamide; TRIS, 3-(tris(trimethylsilyloxy)silyl) propyl methacrylate; NVC, $N$-vinylcarbazole; VneoD, Vinyl neo-Decanoate; VAc, vinyl acetate; MAN, methacrylonitrile; MAA, methacrylic acid; AN, acrylonitrile; EEMA, 2-ethoxyethyl methacrylate; PEGEEMA, poly(ethylene glycol) ethyl ether methacrylate; CHMA, cyclohexyl methacrylate; GMA, glycidyl methacrylate; BzMA, benzyl methacrylate; iBoMA, isobornyl methacrulate; PMOS, $p$-methoxystyrene; PMeS, p-methylstyrene; PFS, p-fluorostyrene; PClS, p-chlorostyrene; PBrS, p-bromostyrene.

Nevertheless, many important kinetic steps are not yet accessible by experiments, or at least an accurate and sufficiently reliable determination of the corresponding rate coefficients is not yet achievable. For instance, the kinetic characterization of secondary reactions such as backbiting, MCR propagation, $\beta$-scission, and intermolecular chain transfers is often not a trivial task, especially concerning some novel and attractive acrylate polymer systems involving fully or partially ionized species. Even the simple propagation step turns out to be a difficult one to deal with if it is inserted in the context of copolymerization; indeed, no direct access to cross-propagation reaction kinetics can be obtained experimentally.

\section{Quantum Chemistry}

The development of computational chemistry and specifically of the advanced methods based on quantum mechanics (QM) has provided a powerful alternative approach to the investigation of reaction kinetics in FRP as reported in literature by Coote and Lin [45]. Computational methods can be applied to the study of individual reactions without the limitations of an experimental investigation, such as restricted ranges of temperature operability and toxicity of the materials. Moreover, with the use of QC one can focus on individual reaction pathways, which are not directly accessible by experiments. In this way, the complex kinetic scheme of FRP can be investigated with a high level of detail; the relevance of very specific reactions and their contribution in the polymerization mechanism can be understood and quantified.

Nowadays, a plethora of commercial and academic software packages can be adopted to perform QC calculations. Most common commercial ones include amsterdam density functional program package (ADF) [46], general atomic and molecular electronic structure system (GAMESS) [47], Gaussian [48], 
Jaguar [49], MOLCAS [50], MOLPRO [51], Q-CHEM [52], Spartan [53], and Turbomole [54], while software packages such as advanced concepts in electronic structure (ACES) [55], BigDFT [56,57], $\mathrm{CP} 2 \mathrm{~K}$ [58], and FreeON [59] are free licensed. All of the reported software packages include the Hartree-Fock (HF) method [60] as well as the density functional theory (DFT) [61], which are the most widely adopted ones in the computational modeling of FRP kinetics.

QM approaches have reached a good level of reliability through a series of studies where the reported methods were applied to the kinetics investigation of several FRP systems and the obtained results were then compared to experimental data, whenever they were available. It is worth mentioning that a better description of reactive systems can be achieved by applying a higher level of theory calculations. Unfortunately, computer power is not yet sufficient to treat system with a very large number of electrons as it happens for polymers. Accordingly, the use of composite methods that can be implemented to treat different portion of the molecule at different levels of theory is proposed [45]. In many cases, the provided accuracy was found to be satisfactory compared to experimental uncertainties, although it is worth noting that more convincing results have been obtained so far for the reactivity ratios rather than for the absolute kinetic coefficients.

QM approaches are adopted to accurately determine molecular geometries (bond lengths, bond angles, and torsional angles). Starting from these data, frequencies of molecular species, energetic reaction profiles, vibrational transition state structures, and reaction frequency factors can be obtained explicitly and directly [62-66]. The rate constant of reactions involved in a FRP system can be expressed through the Arrhenius equation. Two parameters are then required to determine the kinetic constant: the frequency factor, $A$, and the activation energy, $E_{\mathrm{a}}$. These parameters are evaluated through the classic transition state theory (TST) as:

$$
k(T)=A e^{\left(\frac{-E_{\mathrm{a}}}{\mathrm{k}_{\mathrm{b}} T}\right)}=\frac{\mathrm{k}_{\mathrm{b}} T}{\mathrm{~h}} \frac{Q^{\neq}}{Q^{\mathrm{R}}} e^{\left(\frac{-E_{\mathrm{a}}}{\mathrm{k}_{\mathrm{b}} T}\right)}
$$

where $\mathrm{k}_{\mathrm{b}}$ and $\mathrm{h}$ are the Boltzmann and Plank constants, respectively; in addition, $T$ is the temperature, $E_{\mathrm{a}}$ is the activation energy of the process calculated from the difference between the electronic energy of the transition state (TS) and the energy of the reactants (including zero-point energy), and $Q$ represents the product of the partition functions $\left(q^{\text {trans }}, q^{\mathrm{vib}}, q^{\text {rot }}\right.$ and $\left.q^{\mathrm{el}}\right)$ for the transition state $\left(^{\ddagger}\right)$ and reactants $\left({ }^{\mathrm{R}}\right)$. In particular, $q^{\mathrm{el}}$ is the electronic partition function while $q^{\text {trans }}, q^{\text {vib }}$ and $q^{\text {rot }}$ are the translational, vibrational, and rotational partition functions, respectively, calculated according to the following equations:

$$
\begin{gathered}
q^{\text {trans }}=\frac{\left(2 \pi m \mathrm{k}_{\mathrm{b}} T\right)^{\frac{3}{2}}}{\mathrm{~h}^{3}} V \\
q^{\text {vib }}=\prod_{i=1}^{N_{\text {vib }}} \frac{1}{1-\exp \left(-\frac{\mathrm{h} v_{\mathrm{i}}}{\mathrm{k}_{\mathrm{b}} T}\right)} \\
q^{\text {rot }}=\frac{8 \pi^{2}\left(2 \pi \mathrm{k}_{\mathrm{b}} T\right)^{\frac{3}{2}} \sqrt{\mathrm{I}_{\mathrm{x}} \mathrm{I}_{\mathrm{y}} \mathrm{I}_{\mathrm{z}}}}{\sigma \mathrm{h}^{3}}
\end{gathered}
$$

where $V$ is the volume, $m$ the particle mass, $v_{\mathrm{i}}$ the vibrational frequency, $\mathrm{I}_{\mathrm{x}} \mathrm{I}_{\mathrm{y}} \mathrm{I}_{\mathrm{z}}$ the product of the three rotational constants, and $\sigma$ the rotational symmetry number [67-69].

In general, geometries can be predicted with a high accuracy. The estimation of the activation energy suffers from uncertainty that is commonly assumed to be within $4 \mathrm{~kJ} / \mathrm{mol}$, while the larger inaccuracy 
is found in the determination of the pre-exponential factor $[45,70]$. That is the reason why QC better estimates reactivity ratios, for which systematic errors are substantially decreased.

The computational results of the most relevant kinetic investigations on FRP that have been performed so far using QM methods are discussed hereinafter. Computational approaches were first applied to the kinetic characterization of propagation reactions of small radicals, and then progressively moved towards the investigation of larger molecules [71,72]. The development of cost-effective methods, such as those based on the DFT, in combination with the increasing power of the calculators has made possible of scale-up, which was required to investigate chain-length dependency of rate coefficients and lately the reactivity of MCRs. Therefore, the study of ultimate and penultimate unit effects in copolymer propagation as well as of a large variety of chain-transfer reaction was made feasible. The body of the review is structured by dedicating the sections to specific classes of reactions (i.e., initiation and propagation, propagation in copolymerization, and secondary reactions), according to how they have been chronologically approached by QC investigations, trying to critically evaluate reported results, assisting for a future development.

\section{From Initiation to Propagation}

Several $a b$ initio studies were dedicated to the investigation of Arrhenius parameters for the reactions of radical addition to the double bond in the late 1980s and the beginning of 1990s. The addition of a methyl radical to various moieties was first studied [73-75]. Later, the addition of larger radicals to alkenes was investigated, clarifying whether an enthalpy or a polar effect governs the radical reactivity [76]. These preliminary studies proved that the obtained results greatly depend on the selected computational method.

In the mid-1990s, great efforts were made to clarify which computational methods were best suited to the estimation of the Arrhenius parameters of radical addition to alkenes [65,77,78]. Different levels of theory were applied on various propagation reactions and a suitable approach for the prediction of energy barrier was proposed. In these studies, it was proposed that the penultimate unit could significantly affect the estimated values of the frequency factor for radical addition reactions. Some preliminary QC studies examined the presence of the penultimate unit effect on the computational predictions in the radical polymerization field [79-81].

All of the studies reported previously paved the way for the elaboration of the milestone study by Fischer and Radom, who studied the factors that control the addition of various carbon-centered radicals to alkenes [62]. In this study, they found that the predictions of the rate coefficients are more sensitive to the choice of the theoretical procedure than to the choice of the basis set. It was also demonstrated that there is a direct correlation between the applied level of theory used and the accuracy of the obtained results. In particular, as the level of theory increases, the deviation of computational results from experimental values decreases.

As anticipated, the development of the computational methods and the increase of the computing power enabled the study of addition reactions for larger radicals $[68,82,83]$. Therefore, the computational studies were moved from the radical initiation-like reactions discussed above to the true propagation reactions in FRP. The first computational studies of propagation reactions of FRP have been performed for homopolymer system of small molecules. In particular, propagation reaction of alkenes that are 
characterized by very simple molecular structures such as ethene [84,85], vinyl chloride [86,87], and acrylonitrile [86-88] were studied. Gradually, the interest was turned to the study of homopolymer systems of more complex monomers such as styrene and various acrylates [66,89] and to that of monomers with larger substituent [88,90] such as $\alpha$-substituted acrylates [91].

From the study of the aforementioned monomers with large substituent groups arose the need for the structural study of the reactants and the products of the propagation reactions. The presence of large or structurally complex substituent groups increases the number of the rotational degrees of freedom of the moieties, a factor that can significantly affect the reactivity of the radicals. Several studies have been focused to highlight the correlation between the geometries of the radicals and their reactivity [92-94].

In addition, the presence of chain length effect and intramolecular interactions were investigated through QC simulations for simple homopolymer systems [91,93,95]. The investigation of these issues needs to be addressed in order to estimate with accuracy the propagation reaction rates. Computational results obtained for the propagation reaction rate coefficients for the homopolymerization of various monomers are presented in Table 2.

Table 2. Propagation rate coefficients, $k$, and Arrhenius parameters (activation energy, $E_{\mathrm{a}}$, and pre-exponential factor, $A$ ) of the free radical polymerization of various monomers estimated through quantum chemistry (QC) simulations.

\begin{tabular}{|c|c|c|c|c|c|c|}
\hline Monomer & Method & Year & $\begin{array}{c}E_{\mathrm{a}} \\
(\mathrm{kJJ} / \mathrm{mol})\end{array}$ & $\begin{array}{l}\lg (A)(A \\
\mathrm{L} / \mathrm{mol} / \mathrm{s})\end{array}$ & $\begin{array}{c}k\left(50{ }^{\circ} \mathrm{C}\right. \\
\mathrm{L} / \mathrm{mol} / \mathrm{s})\end{array}$ & Reference \\
\hline \multirow{3}{*}{ St } & B3LYP/6-31G(d,p) & 2011 & 34.7 & 8.42 & $6.43 \times 10^{2}$ & [96] \\
\hline & $\begin{array}{c}\text { BP86/cc-PVTZ } \\
\text { (corrected with CCSD) }\end{array}$ & 2009 & 41.5 & 6.57 & $7.22 \times 10^{-1}$ & [97] \\
\hline & $\begin{array}{l}\text { B3LYP/6-311+G(d,p)// } \\
\text { B3LYP/6-31G(d,p) }\end{array}$ & 2009 & 34.6 & 8.25 & $4.51 \times 10^{2}$ & [98] \\
\hline \multirow{5}{*}{ MA } & B3LYP/6-31G(d,p) & 2011 & 27.4 & 8.55 & $1.31 \times 10^{4}$ & [96] \\
\hline & $\begin{array}{l}\text { G3(MP2)-RAD// } \\
\text { B3LYP/6-31G(d) }\end{array}$ & 2010 & 21.8 & 7.50 & $9.43 \times 10^{3}$ & [99] \\
\hline & $\begin{array}{c}\text { BP86/cc-PVTZ } \\
\text { (corrected with CCSD) }\end{array}$ & 2009 & 23.8 & 6.14 & $1.95 \times 10^{2}$ & [97] \\
\hline & $\begin{array}{c}\text { MPWB1K/6-311+G(3df,2p)// } \\
\text { B3LYP//6-31+G(d) }\end{array}$ & 2009 & 24.3 & 6.52 & $3.89 \times 10^{2}$ & [91] \\
\hline & $\begin{array}{l}\text { MPWB1K/6-31G(d,p)// } \\
\text { B3LYP//6-31G(d) }\end{array}$ & 2008 & 21.5 & 7.78 & $2.01 \times 10^{4}$ & [89] \\
\hline $\mathrm{BA}$ & B3LYP/6-31G(d,p) & 2011 & 26.1 & 8.36 & $1.38 \times 10^{4}$ & [96] \\
\hline EA & B3LYP/6-31G(d,p) & 2011 & 27.0 & 8.44 & $1.18 \times 1010^{4}$ & [96] \\
\hline HEA & B3LYP/6-31G(d,p) & 2012 & 22.5 & 7.66 & $1.05 \times 10^{4}$ & [24] \\
\hline \multirow{3}{*}{ MMA } & $\begin{array}{l}\text { MPWB1K/6-31G(d,p)// } \\
\text { B3LYP//6-31G(d,p) }\end{array}$ & 2015 & 11.1 & 4.38 & $3.84 \times 10^{2}$ & {$[100]$} \\
\hline & B3LYP/6-31G(d,p) & 2011 & 33.1 & 8.01 & $4.54 \times 10^{2}$ & [96] \\
\hline & $\begin{array}{c}\text { BP86/cc-PVTZ } \\
\text { (corrected with CCSD) }\end{array}$ & 2009 & 26.0 & 6.06 & $7.16 \times 10$ & [97] \\
\hline
\end{tabular}


Table 2. Cont.

\begin{tabular}{|c|c|c|c|c|c|c|}
\hline Monomer & Method & Year & $\begin{array}{c}E_{\mathrm{a}} \\
(\mathrm{kJ} / \mathrm{mol})\end{array}$ & $\begin{array}{l}\lg (A)(A, \\
\mathrm{L} / \mathrm{mol} / \mathrm{s})\end{array}$ & $\begin{array}{c}k\left(50{ }^{\circ} \mathrm{C}\right. \\
\mathrm{L} / \mathrm{mol} / \mathrm{s})\end{array}$ & Reference \\
\hline \multirow[t]{2}{*}{ MMA } & $\begin{array}{l}\text { B3LYP/6-311+G(d,p)// } \\
\text { B3LYP/6-31G(d,p) }\end{array}$ & 2009 & 26.0 & 7.10 & $7.86 \times 10^{2}$ & [98] \\
\hline & $\begin{array}{l}\text { MPWB1K/6-31G(d,p)// } \\
\text { B3LYP//6-31G(d) }\end{array}$ & 2008 & 22.7 & 6.19 & $3.30 \times 10^{2}$ & [89] \\
\hline BMA & $\begin{array}{c}\text { B3LYP/6-311+G(d,p)// } \\
\text { B3LYP/6-31G(d,p) }\end{array}$ & 2009 & 26.5 & 7.57 & $1.92 \times 10^{3}$ & [98] \\
\hline GMA & $\begin{array}{l}\text { B3LYP/6-311+G(d,p)// } \\
\text { B3LYP/6-31G(d,p) }\end{array}$ & 2009 & 27.5 & 7.45 & $1.01 \times 10^{3}$ & [98] \\
\hline HEMA & $\begin{array}{l}\text { B3LYP/6-311+G(d,p)// } \\
\text { B3LYP/6-31G(d,p) }\end{array}$ & 2009 & 26.8 & 7.10 & $5.83 \times 10^{2}$ & [98] \\
\hline \multirow{3}{*}{ VAc } & B3LYP/6-311+G(d,p) & 2010 & 29.7 & 7.33 & $3.36 \times 10^{2}$ & [101] \\
\hline & $\begin{array}{l}\text { G3(MP2)-RAD// } \\
\text { B3LYP/6-31G(d) }\end{array}$ & 2010 & 17.6 & 6.10 & $1.79 \times 10^{3}$ & [99] \\
\hline & $\begin{array}{c}\text { BP86/cc-PVTZ } \\
\text { (corrected with CCSD) }\end{array}$ & 2009 & 23.9 & 6.61 & $5.56 \times 10^{2}$ & [97] \\
\hline VDF & $\begin{array}{l}\text { MPWB1K/6-31G(d,p)// } \\
\text { B3LYP/6-31G(d,p) }\end{array}$ & 2014 & 20.5 & 6.62 & $2.02 \times 10^{3}$ & [102] \\
\hline HFP & $\begin{array}{l}\text { MPWB1K/6-31G(d,p)// } \\
\text { B3LYP/6-31G(d,p) }\end{array}$ & 2014 & 12.6 & 3.85 & $6.49 \times 10$ & [102] \\
\hline TFE & $\begin{array}{l}\text { MPWB1K/6-31G(d,p)// } \\
\text { B3LYP/6-31G(d,p) }\end{array}$ & 2014 & 10.6 & 6.14 & $2.67 \times 10^{4}$ & [102] \\
\hline $\mathrm{BCA}$ & $\begin{array}{l}\text { MPWB1K/6-31G(d,p)// } \\
\text { B3LYP//6-31G(d,p) }\end{array}$ & 2015 & 17.5 & 3.95 & $1.32 \times 10$ & [100] \\
\hline \multirow[t]{2}{*}{ AA } & $\begin{array}{c}\text { BP86/cc-PVTZ } \\
\text { (corrected with CCSD) }\end{array}$ & 2009 & 24.2 & 6.29 & $2.38 \times 10^{2}$ & [97] \\
\hline & B3LYP/6-31+G(d) & 2004 & 23.3 & 7.57 & $6.34 \times 10^{3}$ & [103] \\
\hline MAA & $\begin{array}{c}\text { BP86/cc-PVTZ } \\
\text { (corrected with CCSD) }\end{array}$ & 2009 & 29.0 & 6.17 & $3.02 \times 10$ & [97] \\
\hline NVP & $\begin{array}{c}\text { BP86/cc-PVTZ } \\
\text { (corrected with CCSD) }\end{array}$ & 2009 & 33.2 & 6.74 & $2.35 \times 10$ & [97] \\
\hline \multirow{2}{*}{ AN } & B3LYP/6-31G(d,p) & 2011 & 31.4 & 8.74 & $4.59 \times 10^{3}$ & [96] \\
\hline & $\begin{array}{l}\text { G3(MP2)-RAD// } \\
\text { B3LYP/6-31G(d) }\end{array}$ & 2006 & 22.4 & 6.40 & $5.99 \times 10^{2}$ & [86] \\
\hline EHMA & $\begin{array}{c}\text { MPWB1K/6-311+G(3df,2p)// } \\
\text { B3LYP/6-31+G(d) }\end{array}$ & 2012 & 24.4 & 5.26 & $2.06 \times 10$ & [93] \\
\hline \multirow{4}{*}{$\mathrm{VC}$} & $\begin{array}{c}\text { MPWB1K/6-31G(d,p)// } \\
\text { B3LYP/6-31G(d,p) }\end{array}$ & 2012 & 23.5 & 7.95 & $1.41 \times 10^{4}$ & [104] \\
\hline & B3LYP/6-31G(d,p) & 2011 & 23.7 & 9.03 & $1.57 \times 10^{5}$ & [105] \\
\hline & $\begin{array}{l}\text { BMK/6-311+G(3df,2p)// } \\
\text { B3LYP/6-31G(d) }\end{array}$ & 2007 & 32.7 & 3.90 & $4.09 \times 10^{-2}$ & [106] \\
\hline & $\begin{array}{l}\text { G3(MP2)-RAD// } \\
\text { B3LYP/6-31G(d) }\end{array}$ & 2006 & 17.2 & 6.59 & $6.43 \times 10^{3}$ & [86] \\
\hline
\end{tabular}

St, styrene; MA, methyl acrylate; BA, Butyl acrylate; EA, ethyl acrylate; MMA, methyl methacrylate; EMA, ethyl methacrylate; BMA, butyl methacrylate; HEA, hydroxyethyl acrylate; HEMA, 2-hydroxyethyl methacrylate; VAc, vinyl acetate; VDF, vinylidene fluoride; HFP, hexafluoropropylene; TFE, tetrafluoroethylene; BCA, butyl cyanoacrylate; AA, acrylic acid; AN, acrylonitrile; GMA, glycidyl methacrylate; EHMA, ethyl a-hydroxy methacrylate; VC, vinyl chloride; NVP, $N$-vinylpyrrolidone. 
A comparison between computational and experimental results for the estimation of propagation kinetics is provided in Figure 1. For each homopolymer system, the relative distance between experimental and computational predictions of activation energy, pre-exponential factor, and rate constant are reported. It should be noted that only the systems for which both computational and experimental data were available are reported in Figure 1.
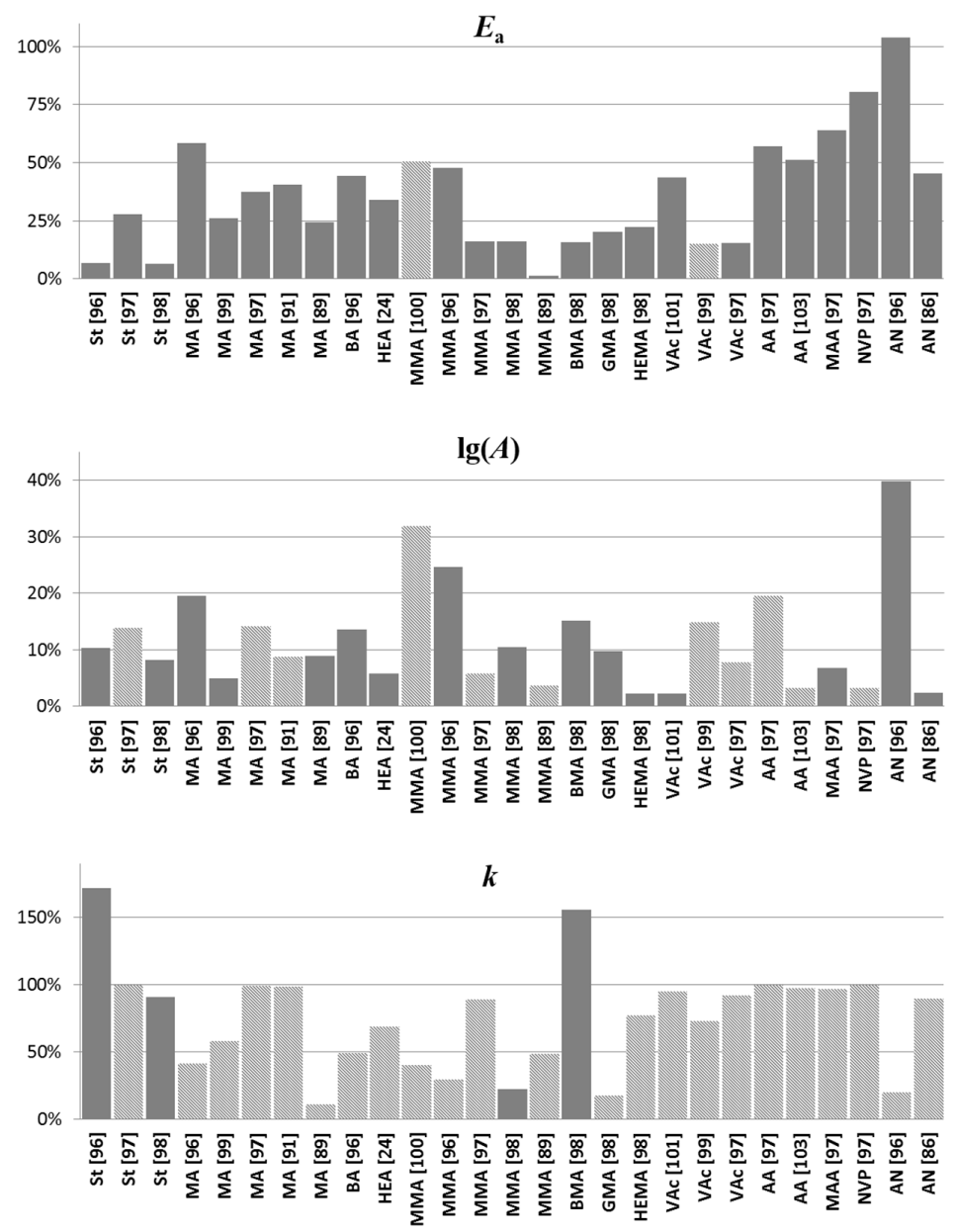

Figure 1. Mean unsigned error between experimental and computational results ( $c f$. data in Tables 1 and 2 respectively) of activation energy, $E_{\mathrm{a}}$, pre-exponential factor, $\lg (A)$, and rate constant at $50{ }^{\circ} \mathrm{C}, k$, for the propagation reactions of various FRP systems. For each column, the number between square brackets indicates the literature source of the computational value adopted, with reference to the data in Table 2. The QC data either overestimate (filled columns) or underestimate (striped columns) the corresponding experimental parameters.

\section{Copolymerization}

The development of computational approaches suited to accurately investigate the addition reactions of large radicals and the chain length effect directed interest to copolymer systems. Reactions that could be in principle considered as copolymerization were first studied in the early 2000s [68]. However, the first complete computational study of a copolymer system was reported only a couple of years later. Namely, the copolymerization of ethylene with vinyl acetate was studied extensively and the kinetics of all the propagation reactions involved in its FRP process were characterized [107]. 
Since then, many copolymer systems of common acrylates and methacrylates, styrene, and functional acrylates as well as of other common monomers were studied applying both the terminal model (TM) [108] and the penultimate unit effect (PUE) [109,110] model [101,107,111-115], which are detailed in Figure 2. A list of relevant computational results for the copolymer systems studied by the application of QM methods is presented in Table 3. Whenever available, experimental results for the same systems are listed as well.

The study of copolymerization systems via QC leads to a better estimation of kinetic parameters compared with the study of homo-propagation reactions. As anticipated in the Quantum Chemistry Section, reactivity ratio are less affected by systematic errors and uncertainties are mutually eliminated allowing a good evaluation of kinetic parameters of primary importance in the polymer science, as is the case of the parameters reported in Table 3. Due to the huge number of possible copolymer systems of interest and the effort that has to be devoted for the experimental determination of kinetics in copolymer systems, QC represents a powerful tool for the study of copolymer reaction engineering.

$$
\begin{aligned}
& \text { Terminal model } \\
& R M_{\mathrm{i}}^{*}+M_{\mathrm{j}} \stackrel{k_{p, i j}}{\longrightarrow} R M_{\mathrm{i}} M_{\mathrm{j}}^{*} \quad i, j=1 \text { or } 2 \\
& r_{i}=\frac{k_{p, i i}}{k_{p, i j}} \\
& F_{i}^{i n s t}=\frac{r_{i} f_{i}^{2}+f_{i} f_{j}}{r_{i} f_{i}^{2}+2 f_{i} f_{j}+r_{i} f_{j}^{2}} \quad \succ i \neq j \text { and } i, j=1 \text { or } 2 \\
& k_{p, \text { cop }}=\frac{r_{i} f_{i}^{2}+2 f_{i} f_{j}+r_{j} f_{j}^{2}}{\left(\frac{r_{i} f_{i}}{k_{p, i i}}\right)+\left(\frac{r_{j} f_{j}}{k_{p, j j}}\right)} \\
& \text { Penultimate unit model } \\
& R M_{\mathrm{i}} M_{\mathrm{j}}^{*}+M_{\mathrm{k}} \stackrel{k_{p, i j k}}{\longrightarrow} R M_{\mathrm{i}} M_{\mathrm{j}} M_{\mathrm{k}}^{*} \quad i, j, k=1 \text { or } 2 \\
& r_{i}=\frac{k_{p, i i i}}{k_{p, i i j}} \quad s_{i}=\frac{k_{p, j i i}}{k_{p, i i i}} \quad r_{i}^{\prime}=\frac{k_{p, j i i}}{k_{p, i i j}} \\
& \overline{r_{i}}=r_{i}^{\prime}\left(\frac{f_{i} r_{i}+f_{j}}{f_{i} r_{i}^{\prime}+f_{j}}\right) \quad \overline{k_{p, i i}}=k_{p, i i i}\left(\frac{f_{i} r_{i}+f_{j}}{f_{i} r_{i}+\frac{f_{j}}{s_{i}}}\right) \\
& F_{i}^{i n s t}=\frac{\bar{r}_{i} f_{i}^{2}+f_{i} f_{j}}{\bar{r}_{i} f_{i}^{2}+2 f_{i} f_{j}+\bar{r}_{j} f_{j}^{2}} \quad k_{p, c o p}=\frac{\bar{r}_{i} f_{i}^{2}+2 f_{i} f_{j}+\bar{r}_{j} f_{j}^{2}}{\left(\frac{\bar{r}_{i} f_{i}}{k_{p, i i}}\right)+\left(\frac{\overline{r_{j}} f_{j}}{k_{p, j j}}\right)} \\
& \text { Implicit penultimate unit model: } \quad \overline{r_{i}}=r_{i}=\frac{k_{p, i i}}{k_{p, i j}} \quad i \neq j \text { and } i, j=1 \text { or } 2
\end{aligned}
$$

Figure 2. Definition of reactivity ratios and fundamental equations of the terminal and the penultimate unit models of copolymerization for a binary system. $R M_{\mathrm{i}}{ }^{*}$, radical with terminal monomer unit of type $i ; M_{\mathrm{j}}$, monomer of type $j ; k$, propagation rate coefficient; $r$, monomer reactivity ratio; $F$, instantaneous copolymer composition; $f$, composition of the monomer phase; $R M_{\mathrm{i}} M_{\mathrm{j}}{ }^{*}$, radical with terminal monomer unit of type $j$ and penultimate unit of type $i ; s$, radical reactivity ratio. 
Table 3. Parameter values of the reactivity rations of various copolymer systems estimated by QC and comparison with experimental data.

\begin{tabular}{|c|c|c|c|c|c|c|c|c|c|c|}
\hline \multirow{2}{*}{$\begin{array}{l}\text { Copolymer } \\
\text { System }\end{array}$} & \multicolumn{6}{|c|}{ Computational } & \multicolumn{4}{|c|}{ Experimental } \\
\hline & Method & $\begin{array}{c}\text { Copolymerization } \\
\text { Model }\end{array}$ & $\begin{array}{c}T \\
\left({ }^{\circ} \mathbf{C}\right)\end{array}$ & Year & $\begin{array}{c}\text { Reactivity } \\
\text { Ratios/Propagation Rate } \\
\text { Coefficients }(\mathrm{L} / \mathrm{mol} / \mathrm{s})\end{array}$ & Reference & $\begin{array}{c}T \\
\left({ }^{\circ} \mathbf{C}\right)\end{array}$ & Year & $\begin{array}{c}\text { Reactivity } \\
\text { Ratios/Propagation Rate } \\
\text { Coefficients }(\mathrm{L} / \mathrm{mol} / \mathrm{s})\end{array}$ & Reference \\
\hline \multirow[t]{2}{*}{ ST/MMA } & \multirow[t]{2}{*}{ B3LYP/6-31G(d,p) } & TM, IPUE & 40 & 2011 & $\begin{array}{c}r_{1}=0.53 ; r_{2}=0.68 \\
s_{1}=0.23 ; s_{2}=0.99\end{array}$ & [96] & 40 & 1985 & $\begin{array}{c}r_{1}=0.52 ; r_{2}=0.46 \\
s_{1}=0.30 ; s_{2}=0.53\end{array}$ & [110] \\
\hline & & $\mathrm{TM}$ & 100 & 2009 & $k_{\mathrm{p}, \mathrm{cop}}=5.05 \times 10^{3}$ & [98] & 20 & 1997 & $r_{1}=0.39 ; r_{2}=0.51$ & [116] \\
\hline \multirow{3}{*}{ ST/HEMA } & B3LYP/6-31G(d,p) & $\mathrm{TM}$ & 100 & 2012 & $r_{1}=0.20 ; r_{2}=0.51$ & [114] & \multirow{2}{*}{50} & \multirow{2}{*}{2009} & \multirow{2}{*}{$\begin{array}{c}r_{1}=0.27 ; r_{2}=0.49 \\
s_{1}=0.38 ; s_{2}=1.34\end{array}$} & \multirow{2}{*}{ [98] } \\
\hline & M06-2X/6-31G(d,p) & $\mathrm{TM}$ & 25 & 2014 & $r_{1}=0.05 ; r_{2}=0.65$ & [117] & & & & \\
\hline & B3LYP/6-31G(d,p) & $\mathrm{TM}$ & 100 & 2009 & $k_{\mathrm{p}, \mathrm{cop}}=12.37 \times 10^{3}$ & {$[98]$} & 70 & 1998 & $k_{\mathrm{p}, \mathrm{cop}}=4.11 \times 10^{3}$ & {$[25]$} \\
\hline \multirow[t]{2}{*}{ ST/GMA } & \multirow[t]{2}{*}{ B3LYP/6-31G(d,p) } & $\mathrm{TM}$ & 100 & 2012 & $r_{1}=0.34 ; r_{2}=0.56$ & [114] & $50-160$ & 2008 & $\begin{array}{c}r_{1}=0.31 ; r_{2}=0.51 \\
s_{1}=0.28 ; s_{2}=1.05\end{array}$ & [118] \\
\hline & & $\mathrm{TM}$ & 100 & 2009 & $k_{\mathrm{p}, \mathrm{cop}}=7.37 \times 10^{3}$ & {$[98]$} & 70 & 2008 & $k_{\mathrm{p}, \mathrm{cop}}=1.64 \times 10^{3}$ & {$[118]$} \\
\hline \multirow[t]{2}{*}{ ST/BMA } & \multirow[t]{2}{*}{ B3LYP/6-31G(d,p) } & $\mathrm{TM}$ & 100 & 2012 & $r_{1}=0.46 ; r_{2}=0.60$ & [114] & $50-150$ & 2006 & $\begin{array}{c}r_{1}=0.61 ; r_{2}=0.42 \\
s_{1}=0.44 ; s_{2}=0.62\end{array}$ & [119] \\
\hline & & $\mathrm{TM}$ & 100 & 2009 & $k_{\mathrm{p}, \mathrm{cop}}=5.39 \times 10^{3}$ & [98] & $70-140$ & 2006 & $k_{\mathrm{p}, \mathrm{cop}}=1.23 \times 10^{3}$ & [119] \\
\hline \multirow{2}{*}{ ST/HEA } & \multirow{2}{*}{ B3LYP/6-31G(d,p) } & $\mathrm{TM}$ & 50 & 2011 & $r_{1}=0.31 ; r_{2}=0.14$ & [120] & 60 & 1998 & $r_{1}=0.46 ; r_{2}=0.20$ & [121] \\
\hline & & EPUE & 50 & 2012 & $\begin{array}{c}r_{11}=0.72 ; r_{21}=0.57 ; \\
r_{12}=0.38 ; r_{22}=0.62 ; \\
s_{1}=0.72 ; s_{2}=3.01\end{array}$ & {$[24]$} & 50 & 2012 & $\begin{array}{c}r_{1}=0.44 ; r_{2}=0.18 \\
s_{1}=3.00 ; r_{1}=0.91\end{array}$ & {$[24]$} \\
\hline ST/MA & B3LYP/6-31G(d,p) & $\mathrm{TM}$ & 25 & 2011 & $r_{1}=0.22 ; r_{2}=0.11$ & {$[82]$} & 25 & 1991 & $r_{1}=0.73 ; r_{2}=0.19$ & {$[122]$} \\
\hline \multirow{5}{*}{ MMA/MA } & MPWB1K/6-31G(d,p) & TM, IPUE & 23 & 2008 & $\begin{array}{c}r_{1}=2.99 ; r_{2}=0.38 \\
s_{1}=1.71 ; r_{1}=0.39\end{array}$ & [115] & \multirow{5}{*}{50} & \multirow{5}{*}{1971} & \multirow{5}{*}{$r_{1}=2.23 ; r_{2}=0.36$} & \multirow{5}{*}[123]{} \\
\hline & & EPUE & 23 & 2008 & $\begin{array}{r}r_{11}=2.81 ; r_{21}=3.17 ; \\
r_{12}=0.38 ; r_{22}=0.37 ; \\
s_{1}=1.71 ; s_{2}=0.39\end{array}$ & [115] & & & & \\
\hline & B3LYP/6-31G(d) & $\mathrm{TM}$ & 25 & 2006 & $r_{1}=0.83 ; r_{2}=0.49$ & [113] & & & & \\
\hline & B3P86/6-311G(2d,2p) & $\mathrm{TM}$ & 25 & 2006 & $r_{1}=0.97 ; r_{2}=0.41$ & [113] & & & & \\
\hline & B971/6-311G(2d,2p) & TM & 25 & 2006 & $r_{1}=1.06 ; r_{2}=0.37$ & [113] & & & & \\
\hline
\end{tabular}


Table 3. Cont.

\begin{tabular}{|c|c|c|c|c|c|c|c|c|c|c|}
\hline \multirow{2}{*}{$\begin{array}{l}\text { Copolymer } \\
\text { System }\end{array}$} & \multicolumn{6}{|c|}{ Computational } & \multicolumn{4}{|c|}{ Experimental } \\
\hline & Method & $\begin{array}{l}\text { Copolymerization } \\
\text { Model }\end{array}$ & $\begin{array}{c}\boldsymbol{T} \\
\left({ }^{\circ} \mathbf{C}\right)\end{array}$ & Year & $\begin{array}{c}\text { Reactivity } \\
\text { Ratios/Propagation Rate } \\
\text { Coefficients }(\mathrm{L} / \mathrm{mol} / \mathrm{s})\end{array}$ & Reference & $\begin{array}{c}\boldsymbol{T} \\
\left({ }^{\circ} \mathbf{C}\right)\end{array}$ & Year & $\begin{array}{c}\text { Reactivity } \\
\text { Ratios/Propagation Rate } \\
\text { Coefficients }(\mathrm{L} / \mathrm{mol} / \mathrm{s})\end{array}$ & Reference \\
\hline \multirow{3}{*}{ BA/BMA } & B3LYP/6-31G(d) & $\mathrm{TM}$ & 25 & 2006 & $r_{1}=0.44 ; r_{2}=0.84$ & [113] & \multirow{3}{*}{-} & \multirow{3}{*}{-} & \multirow{3}{*}{ - } & \multirow{3}{*}{-} \\
\hline & B3P86/6-311G(2d,2p) & $\mathrm{TM}$ & 25 & 2006 & $r_{1}=0.40 ; r_{2}=0.94$ & [113] & & & & \\
\hline & B971/6-311G(2d,2p) & $\mathrm{TM}$ & 25 & 2006 & $r_{1}=0.36 ; r_{2}=1.06$ & [113] & & & & \\
\hline \multirow[t]{2}{*}{ MA/BA } & B3P86/6-311G(2d,2p) & TM & 25 & 2006 & $r_{1}=1.04 ; r_{2}=0.97$ & [113] & \multirow{2}{*}{-} & \multirow{2}{*}{-} & \multirow{2}{*}{-} & \multirow{2}{*}{-} \\
\hline & B971/6-311G(2d,2p) & $\mathrm{TM}$ & 25 & 2006 & $r_{1}=1.04 ; r_{2}=0.97$ & [113] & & & & \\
\hline \multirow{3}{*}{ MMA/BMA } & B3LYP/6-31G(d) & TM & 25 & 2006 & $r_{1}=0.99 ; r_{2}=1.01$ & [113] & \multirow{3}{*}{20} & \multirow{3}{*}{1988} & \multirow{3}{*}{$r_{1}=0.44 ; r_{2}=0.26$} & \multirow{3}{*}{ [124] } \\
\hline & B3P86/6-311G(2d,2p) & TM & 25 & 2006 & $r_{1}=1.02 ; r_{2}=0.99$ & [113] & & & & \\
\hline & B971/6-311G(2d,2p) & TM & 25 & 2006 & $r_{1}=1.02 ; r_{2}=0.99$ & [113] & & & & \\
\hline \multirow{2}{*}{ MA/MEA } & B3LYP/6-31G(d) & $\mathrm{TM}$ & 25 & 2006 & $r_{1}=0.55 ; r_{2}=0.38$ & [113] & \multirow{2}{*}{-} & \multirow{2}{*}{-} & \multirow{2}{*}{-} & \multirow{2}{*}{-} \\
\hline & B971/6-311G(2d,2p) & TM & 25 & 2006 & $r_{1}=0.53 ; r_{2}=0.42$ & [113] & & & & \\
\hline \multirow{3}{*}{ MMA/MEA } & B3LYP/6-31G(d) & TM & 25 & 2006 & $r_{1}=1.85 ; r_{2}=0.40$ & {$[113]$} & \multirow{3}{*}{-} & \multirow{3}{*}{-} & \multirow{3}{*}{-} & \multirow{3}{*}{-} \\
\hline & B3P86/6-311G(2d,2p) & TM & 25 & 2006 & $r_{1}=2.10 ; r_{2}=0.37$ & {$[113]$} & & & & \\
\hline & B971/6-311G(2d,2p) & TM & 25 & 2006 & $r_{1}=1.96 ; r_{2}=0.43$ & [113] & & & & \\
\hline \multirow{3}{*}{ MA/VAc } & B3LYP/6-31G(d) & $\mathrm{TM}$ & 25 & 2006 & $r_{1}=6.76 ; r_{2}=0.10$ & [113] & \multirow{3}{*}{60} & \multirow{3}{*}{1994} & \multirow{3}{*}{$r_{1}=7.28 ; r_{2}=0.04$} & \multirow{3}{*}{ [125] } \\
\hline & B3P86/6-311G(2d,2p) & TM & 25 & 2006 & $r_{1}=6.77 ; r_{2}=0.10$ & [113] & & & & \\
\hline & B971/6-311G(2d,2p) & $\mathrm{TM}$ & 25 & 2006 & $r_{1}=6.62 ; r_{2}=0.10$ & [113] & & & & \\
\hline
\end{tabular}


Table 3. Cont.

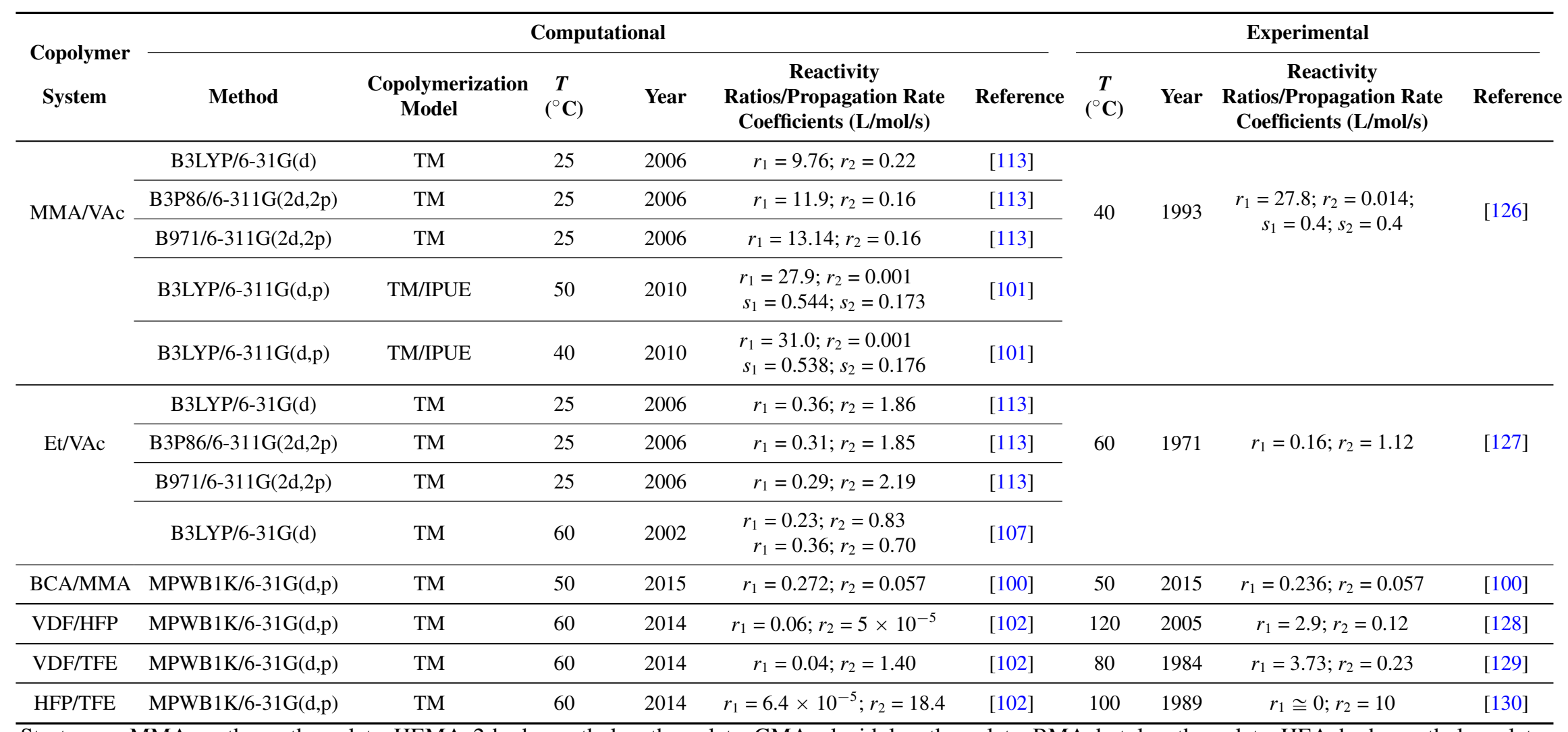

St, styrene; MMA, methy methacrylate; HEMA, 2-hydroxyethyl methacrylate; GMA, glycidyl methacrylate; BMA, butyl methacrylate; HEA, hydroxyethyl acrylate; MA, methyl acrylate; BA, Butyl acrylate; MEA, methyl ethacrylate; Et, Ethylene; VAc, vinyl acetate; BCA, butyl cyanoacrylate; VDF, vinylidene fluoride; HFP, hexafluoropropylene; TFE, tetrafluoroethylene. 
Another challenge relevant to propagation reaction kinetics in FRP on which attention has been focused lately is the solvent effect. Functional monomers and polymers are likely to interact with a polar solvent or reaction medium due to intermolecular forces, which can vary from polarization and dipole interactions to stronger hydrogen bonding. The standard polymerization behavior of the monomers and the radical species can be influenced by these interactions.

From the computational point of view, the solvent effect can be modeled considering explicit solvent molecules and characterizing the specific interactions, or using continuum models [71]. The former approach is clearly more effective, while it is often coupled with a prohibitive computational cost. Few works are reported in the literature in the attempt of investigating the influence of water on the propagation of acrylamide [131], acrylic acid, and methacrylic acid [132], as well as the solvent effect on the tacticity of a propagating MMA chain [133]. One of the major issues of this approach is represented by the restricted number of solvent molecules that can be treated, not to mention that the choice of their displacement around the reaction site that is often unavoidably arbitrary.

Continuum models for treating the solvent effect consider the solvent molecules as implicitly embedded in cavities surrounded by a dielectric continuum. Typical models belonging to this category are COSMO (conductor-like screening model) and PCM (polarizable continuum model), particularly suitable for treating large systems [134,135]. Continuum models are considered adequate for radical thermochemistry, and have been used in the determination of the solvent effect and its relevance for various FRP systems [86,89,99,103]. Encouraging results have been obtained using the quantum mechanics/molecular mechanics (QM/MM) method [132] and the Car-Parrinello molecular dynamics (CPMD) method [136]. Such methods can pave the way to overcome the difficulties in studying a range of solvent-sensitive systems.

\section{Secondary Reactions}

The continuously increasing potential of the QM approaches allowed the extension of the computational studies toward reactions that are lying beyond the fundamental scheme of FRP, the so-called side or secondary reactions. The investigation of secondary reaction kinetics is motivated by two main reasons: on the one hand, the overall process kinetics can be significantly affected by such reactions even under mild reaction conditions; on the other hand, secondary reactions are often responsible for deviations from the desired polymer properties and for the formation of defects in the polymer microstructure.

During the last years many studies were performed with the purpose of investigating the secondary reactions, which are most relevant in FRP processes. Intermolecular hydrogen abstractions like chain transfer to monomer and chain transfer to polymer were studied in various systems [106,137-141]. Intramolecular hydrogen abstractions were investigated as well, focusing on the backbiting reactions that occur when a chain-end radical (CER) abstracts a hydrogen from its own backbone, forming a mid-chain radical (MCR) [70,106,141-144]. Specific attention was paid to the 1:5 backbiting due to its energetically favorable transition state configuration involving a six-atom ring [145]. Chain transfer to small molecules in the reaction mixture like solvent [146,147] or chain transfer agent (CTA) [148,149] were studied in addition to the abstractions of hydrogen atoms from polymer or monomer [150]. 
Computational approaches based on QM were also applied to the determination of MCR reactivity. In particular, the rate coefficients of propagation reaction of MCRs were estimated for various systems $[70,104,142,144]$. Furthermore, both the two symmetric breaks of the carbon-carbon bonds that can occur when a MCR undergoes a $\beta$-scission reaction were studied [70,106,143,144,151,152]. Finally, reactions relevant in high temperature processes like thermal self-initiation were approached by quantum chemical investigations [151,153,154].

In recent years, the quantum tunneling (QT) phenomenon has often been taken into account for the kinetics characterization of reactions where a hydrogen abstraction occurs [155]. According to this phenomenon, a sufficiently small moving particle (i.e., hydrogen radical) can overcome an energy barrier even if its energy is lower than the one required, as it happens in the case of reactions of hydrogen abstraction. QC simulations allow estimating a QT factor, which leads to higher kinetic coefficients of hydrogen abstraction when applied. The quantum tunneling effect is introduced multiplying each rate coefficient evaluated from Equation (1) by the tunneling factor, $Q_{\text {tun, }}$ as shown in Equation (5). This parameter is defined as the ratio between quantum and classical fluxes across the energy barrier, as shown in Equation (6), where $P(E)$ is the transmission probability and $E_{1}$ the activation energy of the forward reaction.

$$
\begin{gathered}
k(T)_{\text {tun }}=k(T) Q_{\text {tun }} \\
Q_{\text {tun }}=\frac{\int_{O}^{\infty} P(E) e^{\left(\frac{-E}{k_{\mathrm{b}} T}\right)} d E}{\int_{E_{1}}^{\infty} e^{\left(\frac{-E}{k_{\mathrm{b}} T}\right)}}
\end{gathered}
$$

The transmission probability can be calculated using the solution of the translational Schrödinger equation as reported in literature $[155,156]$.

The estimated value of the QT factor and, consequently, its effect on the absolute rate coefficient value can be significant within the typical range of temperature of FRP reactions. A selection of the most relevant computationally estimated rate coefficients of secondary reactions in common FRP systems is reported in Table 4, while a schematic representation of some relevant secondary reactions is provided in Figure 3.

The aim of deepening the understanding of FRP mechanisms causes a continuous need for the characterization of reaction kinetics. This process pushes the interest towards increasingly complex and fascinating secondary reactions, such as those involving new types of radicals or originating very specific side-products and defects (e.g., side-backbiting producing short-branch radicals and their following propagation) [70,104]. Their kinetic characterization is still out of the reach of the experimental investigation, so this is the field where QC studies find the widest room for application.

Although in many cases these interesting reactions are part of FRP mechanisms that are not yet clearly well defined, one should not forget the role that QC can take in the improvement of the understanding of such mechanisms by guiding the identification of the most relevant reaction pathways. Especially in the area of secondary reactions, QC can help to clarify how a newly-proposed reaction or pathway can be relevant, thus orienting the investigation of FRP kinetics from the very basis. 
Table 4. Rate coefficients, $k$, and Arrhenius parameters (activation energy, $E_{\mathrm{a}}$, and pre-exponential factor, $A$ ) of secondary reactions of relevant free radical polymerization systems estimated by QC and comparison with the existing data obtained through PLP experiments. The reported rate coefficients are evaluated at the indicated temperatures $(T)$.

\begin{tabular}{|c|c|c|c|c|c|c|c|c|c|c|c|c|c|c|}
\hline \multirow[b]{2}{*}{$\begin{array}{l}\text { Polymer/ } \\
\text { Monomer }\end{array}$} & \multicolumn{9}{|c|}{ Computational } & \multicolumn{5}{|c|}{ Experimental } \\
\hline & Method & $\begin{array}{c}T \\
\left(\mathbf{C}^{\circ}\right)\end{array}$ & Year & $\begin{array}{c}E_{\mathrm{a}} \\
(\mathbf{k J} / \mathbf{m o l})\end{array}$ & $\begin{array}{c}\lg (A)(A, \\
\mathrm{L} / \mathrm{mol} / \mathrm{s} \text { or } \\
1 / \mathrm{s})\end{array}$ & $\begin{array}{c}k(\mathrm{~L} / \mathrm{mol} / \mathrm{s} \text { or } \\
1 / \mathrm{s})\end{array}$ & Reaction & QT & Reference & $\begin{array}{c}\boldsymbol{T} \\
\left({ }^{\circ} \mathbf{C}\right)\end{array}$ & $E_{\mathrm{a}}(\mathbf{k J} \mathbf{J} / \mathbf{m o l})$ & $\begin{array}{c}\lg (A)(A, \\
\mathrm{L} / \mathrm{mol} / \mathrm{s} \\
\text { or } 1 / \mathrm{s})\end{array}$ & $\begin{array}{l}k(\mathrm{~L} / \mathrm{mol} / \mathrm{s} \\
\quad \text { or } 1 / \mathrm{s})\end{array}$ & Reference \\
\hline PEA & $\mathrm{UHF} / 6-31 \mathrm{G}(\mathrm{d})$ & 160 & 2001 & 132.8 & 11.40 & $3.00 \times 10^{-7}$ & $\begin{array}{l}\text { Short-chain } \\
\text { branching }\end{array}$ & - & {$[63]$} & - & - & - & - & - \\
\hline \multirow{5}{*}{ PMA } & $\begin{array}{l}\text { UB3LYP/ } \\
\text { 6-31G(d)// } \\
\text { MPWB1K/ } \\
6-31 G(d, p)\end{array}$ & 100 & 2012 & 57.3 & 12.17 & $1.41 \times 10^{4}$ & Backbiting & No & [145] & - & - & - & - & - \\
\hline & $\begin{array}{c}\text { B3LYP/ } \\
\text { 6-31G(d,p)// } \\
\text { MPWB1K/ } \\
\text { 6-31G(d,p) }\end{array}$ & 100 & 2013 & 50.7 & 10.66 & $4.12 \times 10^{3}$ & Backbiting & Yes & [70] & - & - & - & - & - \\
\hline & $\begin{array}{c}\text { B3LYP/ } \\
\text { 6-31G(d,p)// } \\
\text { MPWB1K/ } \\
\text { 6-31G(d,p) }\end{array}$ & 100 & 2013 & 115.6 & 14.01 & $2.09 \times 10^{-5}$ & $\beta$-Scission & - & {$[70]$} & - & - & - & - & - \\
\hline & $\begin{array}{c}\text { B3LYP/ } \\
\text { 6-31G(d,p) }\end{array}$ & 25 & 2014 & 57.3 & 11.36 & $6.60 \times 10^{2}$ & Backbiting & Yes & [143] & - & - & - & - & - \\
\hline & $\begin{array}{l}\text { B3LYP/ } \\
\text { 6-31G(d) }\end{array}$ & 25 & 2014 & 100.0 & 13.02 & $3.84 \times 10^{-5}$ & $\beta$-Scission & - & [143] & - & - & - & - & - \\
\hline MA & $\begin{array}{l}\text { M06-2X/ } \\
6-31 G(d, p)\end{array}$ & 25 & 2013 & 56.0 & 5.11 & $1.70 \times 10^{-5}$ & $\begin{array}{l}\text { Chain } \\
\text { transfer } \\
\text { to } \\
\text { monomer }\end{array}$ & Yes & [140] & - & - & - & - & - \\
\hline
\end{tabular}


Table 4. Cont.

\begin{tabular}{|c|c|c|c|c|c|c|c|c|c|c|c|c|c|c|}
\hline \multirow[b]{2}{*}{$\begin{array}{l}\text { Polymer/ } \\
\text { Monomer }\end{array}$} & \multicolumn{9}{|c|}{ Computational } & \multicolumn{5}{|c|}{ Experimental } \\
\hline & Method & $\begin{array}{c}T \\
\left(\mathbf{C}^{\circ}\right)\end{array}$ & Year & $\begin{array}{c}E_{\mathrm{a}} \\
(\mathrm{kJ} / \mathrm{mol})\end{array}$ & $\begin{array}{c}\lg (A)(A \\
\mathrm{L} / \mathrm{mol} / \mathrm{s} \text { or } \\
1 / \mathrm{s})\end{array}$ & $\begin{array}{c}k(\mathrm{~L} / \mathrm{mol} / \mathrm{s} \text { or } \\
1 / \mathrm{s})\end{array}$ & Reaction & QT & Reference & $\begin{array}{c}T \\
\left({ }^{\circ} \mathbf{C}\right)\end{array}$ & $E_{\mathrm{a}}(\mathrm{kJ} / \mathbf{m o l})$ & $\begin{array}{c}\lg (A)(A, \\
\mathrm{L} / \mathrm{mol} / \mathrm{s} \\
\text { or } 1 / \mathrm{s})\end{array}$ & $\begin{array}{l}k(\mathrm{~L} / \mathrm{mol} / \mathrm{s} \\
\quad \text { or } 1 / \mathrm{s})\end{array}$ & Reference \\
\hline \multirow[t]{4}{*}{ PBA } & $\begin{array}{l}\text { UB3LYP/ } \\
\text { 6-31G(d)// } \\
\text { MPWB1K/ } \\
\text { 6-31G(d,p) }\end{array}$ & 100 & 2012 & 73.2 & 13.65 & $2.57 \times 10^{3}$ & Backbiting & No & [145] & \multirow[t]{2}{*}{50} & \multirow[t]{2}{*}{33.3} & \multirow[t]{2}{*}{7.97} & \multirow[t]{2}{*}{$3.83 \times 10^{2}$} & \multirow[t]{2}{*}[12,17]{} \\
\hline & $\begin{array}{c}\text { B3LYP/ } \\
\text { 6-31G(d,p)// } \\
\text { MPWB1K/ } \\
\text { 6-31G(d,p) }\end{array}$ & 25 & 2013 & 55.0 & 11.46 & $1.64 \times 10^{3}$ & Backbiting & Yes & [144] & & & & & \\
\hline & $\begin{array}{c}\text { B3LYP/ } \\
\text { 6-31G(d,p)// } \\
\text { MPWB1K/ } \\
\text { 6-31G(d,p) }\end{array}$ & 25 & 2013 & 116.9 & 12.46 & $9.22 \times 10^{-9}$ & $\beta$-Scission & - & [144] & - & - & - & - & - \\
\hline & $\begin{array}{c}\text { B3LYP/ } \\
\text { 6-31G(d,p)// } \\
\text { MPWB1K/ } \\
\text { 6-31G(d,p) }\end{array}$ & 25 & 2014 & 47.1 & 2.72 & $1.09 \times 10^{-4}$ & $\begin{array}{l}\text { Chain } \\
\text { transfer } \\
\text { to } \\
\text { polymer }\end{array}$ & Yes & {$[150]$} & - & - & - & - & - \\
\hline \multirow[t]{2}{*}{ BA } & $\begin{array}{c}\text { B3LYP/ } \\
\text { 6-31G(d,p)// } \\
\text { MPWB1K/ } \\
\text { 6-31G(d,p) }\end{array}$ & 25 & 2014 & 57.4 & 5.24 & $4.03 \times 10^{-4}$ & $\begin{array}{l}\text { Chain } \\
\text { transfer } \\
\text { to } \\
\text { monomer }\end{array}$ & Yes & [150] & \multirow[t]{2}{*}{50} & \multirow[t]{2}{*}{32.6} & \multirow[t]{2}{*}{5.46} & \multirow[t]{2}{*}{1.55} & \multirow[t]{2}{*}{ [157] } \\
\hline & $\begin{array}{l}\text { M06-2X/ } \\
\text { 6-31G(d,p) }\end{array}$ & 25 & 2013 & 31.0 & 5.17 & $5.00 \times 10^{-1}$ & $\begin{array}{l}\text { Chain } \\
\text { transfer } \\
\text { to } \\
\text { monomer }\end{array}$ & Yes & [140] & & & & & \\
\hline
\end{tabular}


Table 4. Cont.

\begin{tabular}{|c|c|c|c|c|c|c|c|c|c|c|c|c|c|c|}
\hline \multirow[b]{2}{*}{$\begin{array}{l}\text { Polymer/ } \\
\text { Monomer }\end{array}$} & \multicolumn{9}{|c|}{ Computational } & \multicolumn{5}{|c|}{ Experimental } \\
\hline & Method & $\begin{array}{c}T \\
\left(\mathbf{C}^{\circ}\right)\end{array}$ & Year & $\begin{array}{c}E_{\mathrm{a}} \\
(\mathrm{kJ} / \mathrm{mol})\end{array}$ & $\begin{array}{c}\lg (A)(A \\
\mathrm{L} / \mathrm{mol} / \mathrm{s} \text { or } \\
1 / \mathrm{s})\end{array}$ & $\begin{array}{c}k(\mathrm{~L} / \mathrm{mol} / \mathrm{s} \text { or } \\
1 / \mathrm{s})\end{array}$ & Reaction & QT & Reference & $\begin{array}{c}T \\
\left({ }^{(} \mathbf{C}\right)\end{array}$ & $E_{\mathrm{a}}(\mathrm{kJ} / \mathbf{m o l})$ & $\begin{array}{c}\lg (A)(A, \\
\mathrm{L} / \mathrm{mol} / \mathrm{s} \\
\text { or } 1 / \mathrm{s})\end{array}$ & $\begin{array}{l}k(\mathrm{~L} / \mathrm{mol} / \mathrm{s} \\
\quad \text { or } 1 / \mathrm{s})\end{array}$ & Reference \\
\hline EA & $\begin{array}{l}\text { M06-2X/ } \\
6-31 G(d, p)\end{array}$ & 25 & 2013 & 41.0 & 5.10 & $9.90 \times 10^{-3}$ & $\begin{array}{l}\text { Chain } \\
\text { transfer } \\
\text { to } \\
\text { monomer }\end{array}$ & Yes & [140] & - & - & - & - & - \\
\hline \multirow[t]{3}{*}{ PVC } & $\begin{array}{c}\text { B3LYP/ } \\
\text { 6-31G(d,p)// } \\
\text { MPWB1K/ } \\
\text { 6-31G(d,p) }\end{array}$ & 57 & 2012 & 74.9 & 14.13 & $1.90 \times 10^{2}$ & Backbiting & Yes & [104] & - & - & - & - & - \\
\hline & $\begin{array}{c}\text { B3LYP/ } \\
\text { 6-31G(d,p)// } \\
\text { MPWB1K/ } \\
\text { 6-31G(d,p) }\end{array}$ & 57 & 2012 & 98.1 & 12.81 & $1.96 \times 10^{-3}$ & $\beta$-Scission & - & [104] & - & - & - & - & - \\
\hline & $\begin{array}{c}\text { B3LYP/ } \\
6-31+\mathrm{G}(\mathrm{d}) / / \\
\mathrm{BMK} / 6-311+ \\
\mathrm{G}(3 \mathrm{fd}, 2 \mathrm{p})\end{array}$ & 57 & 2007 & 72.8 & 11.43 & $8.09 \times 10^{-1}$ & Backbiting & No & [106] & - & - & - & - & - \\
\hline
\end{tabular}

PEA, poly ethyl acrylate; PMA, poly methyl acrylate; MA, methyl acrylate; PBA, poly butyl acrylate; BA, butyl acrylate; EA, ethyl acrylate; PVC, polyvinyl chloride. 
Chain transfer to monomer<smiles>[R]C(=C)CCCCCC#N</smiles>

$$
R_{n}^{*}+M \rightarrow P_{n}+M^{*}
$$

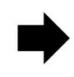<smiles>[2H]CC#N</smiles><smiles>C=C[18OH]</smiles>

Intermolecular chain transfer to polymer

$$
R_{n}^{*}+P_{m} \rightarrow P_{n}+Q_{m}^{*}
$$

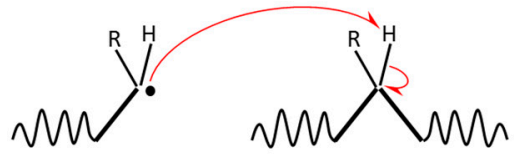<smiles>[2H]CC#N</smiles><smiles>[14CH3][Mg][Mg]</smiles>

Backbiting (intramolecular chain transfer to polymer) $\quad R_{n}^{*} \rightarrow Q_{n}^{*}$

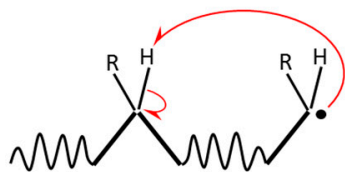<smiles>C1CCCCC1</smiles><smiles>[2H]C([2H])([2H])[14CH3]</smiles>

Chain transfer to agent / solvent (small molecules) $\quad R_{n}^{*}+S \rightarrow P_{n}+S^{*}$<smiles>[2H]C(C#N)CCCNCC</smiles><smiles>[2H]CC#N</smiles>

Propagation on mid-chain radical

$$
Q_{n}^{*}+M \rightarrow R_{n+1}^{*}
$$

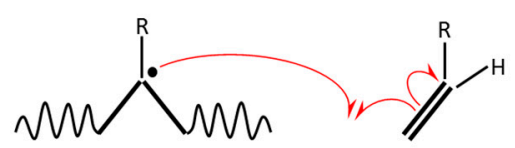

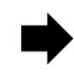<smiles>[R]CC([R])([AlH])CN</smiles>

$\beta$-scission $\quad Q_{n}^{*} \rightarrow P_{n-m}+R_{m}^{*}$

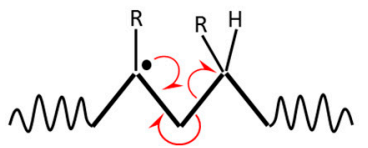<smiles>C1CCCCC1</smiles><smiles>[2H]C(=C)[W]</smiles>

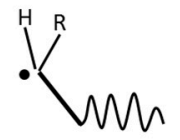

Figure 3. Schematic representation of relevant secondary reactions. $R$, terminal radical; $M$, monomer; $P$, dead polymer; $Q$, mid-chain radical. 


\section{Conclusions}

Computational approaches show great potential for the investigation of many reaction steps of FRP. Nowadays, the computational power of computers allows studying the reactivity of large molecules at high levels of theory. Simultaneously, the continuous development of new methods that can be applied for studying polymer systems at a high level of theory and the control of their reliability through comparison with experimental studies increased the accuracy of the predicted rate coefficients that can be achieved through these approaches.

The use of such a powerful computational tool that is proven to be able to provide accurate predictions for the propagation reactions at FRP can help to overcome some of the experimental issues. In particular, computational approaches can be used for the investigation of the less accessible reactions in FRP. Furthermore, the potential of the approaches is motivation for the investigation of more challenging systems characterized by a high complexity, such as copolymer systems.

In the present review, it was attempted to collect all the computational studies for propagation and secondary reactions during free radical copolymerization. Additionally, experimental values for the same reactions are presented along with the experimental ones. The aim of this work is to provide the reader with a concise, comprehensive, and updated list of the computational studies that dealt with the propagation and the secondary reactions at free radical polymerization.

\section{Conflicts of Interest}

The authors declare no conflict of interest.

\section{References}

1. Cunningham, M.F.; Hutchinson, R.A. Industrial applications and processes. In Handbook of Radical Polymerization; John Wiley \& Sons: Hoboken, NJ, USA, 2003; pp. 333-360.

2. Braun, D. Origins and development of initiation of free radical polymerization processes. Int. J. Polym. Sci. 2009. [CrossRef]

3. Coote, M.L.; Davis, T.P. Copolymerization kinetics. In Handbook of Radical Polymerization; John Wiley \& Sons: Hoboken, NJ, USA, 2003; pp. 263-300.

4. Olaj, O.F.; Bitai, I.; Hinkelmann, F. The laser-flash-initiated polymerization as a tool of evaluating (individual) kinetic constants of free-radical polymerization, 2. The direct determination of the rate-constant of chain propagation. Die Makromol. Chem. 1987, 188, 1689-1702. [CrossRef]

5. Buback, M. Propagation kinetics in radical polymerization studied via pulsed laser techniques. Macromol. Symp. 2009, 275, 90-101. [CrossRef]

6. Barth, J.; Buback, M.; Hesse, P.; Sergeeva, T. Termination and transfer kinetics of butyl acrylate radical polymerization studied via SP-PLP-EPR. Macromolecules 2010, 43, 4023-4031. [CrossRef]

7. Barner-Kowollik, C.; Gunzler, F.; Junkers, T. Pushing the limit: Pulsed laser polymerization of $N$-butyl acrylate at $500 \mathrm{~Hz}$. Macromolecules 2008, 41, 8971-8973. [CrossRef]

8. Beuermann, S.; Buback, M. Rate coefficients of free-radical polymerization deduced from pulsed laser experiments. Prog. Polym. Sci. 2002, 27, 191-254. [CrossRef] 
9. Asua, J.M.; Beuermann, S.; Buback, M.; Castignolles, P.; Charleux, B.; Gilbert, R.G.; Hutchinson, R.A.; Leiza, J.R.; Nikitin, A.N.; Vairon, J.P.; et al. Critically evaluated rate coefficients for free-radical polymerization, 5. Macromol. Chem. Phys. 2004, 205, 2151-2160.

10. Arzamendi, G.; Plessis, C.; Leiza, J.R.; Asua, J.M. Effect of the intramolecular chain transfer to polymer on PLP/SEC experiments of alkyl acrylates. Macromol. Theory Simul. 2003, 12, 315-324. [CrossRef]

11. Reyes, Y.; Arzamendi, G.; Asua, J.M.; Leiza, J.R. Branching at high frequency pulsed laser polymerizations of acrylate monomers. Macromolecules 2011, 44, 3674-3679. [CrossRef]

12. Nikitin, A.N.; Hutchinson, R.A.; Buback, M.; Hesse, P. Determination of intramolecular chain transfer and midchain radical propagation rate coefficients for butyl acrylate by pulsed laser polymerization. Macromolecules 2007, 40, 8631-8641. [CrossRef]

13. Plessis, C.; Arzamendi, G.; Alberdi, J.M.; van Herk, A.M.; Leiza, J.R.; Asua, J.M. Evidence of branching in poly(butyl acrylate) produced in pulsed-laser polymerization experiments. Macromol. Rapid Commun. 2003, 24, 173-177. [CrossRef]

14. Barth, J.; Buback, M.; Hesse, P.; Sergeeva, T. EPR Analysis of $N$-butyl acrylate radical polymerization. Macromol. Rapid Commun. 2009, 30, 1969-1974. [CrossRef] [PubMed]

15. Barth, J.; Buback, M.; Barner-Kowollik, C.; Junkers, T.; Russell, G.T. Single-pulse pulsed laser polymerization-electron paramagnetic resonance investigations into the termination kinetics of $N$-butyl acrylate macromonomers. J. Polym. Sci. Part A 2012, 50, 4740-4748. [CrossRef]

16. Barth, J.; Siegmann, R.; Beuermann, S.; Russell, G.T.; Buback, M. Investigations into chain-length-dependent termination in bulk radical polymerization of $1 \mathrm{H}, 1 \mathrm{H}, 2 \mathrm{H}$, 2H-tridecafluorooctyl methacrylate. Macromol. Chem. Phys. 2012, 213, 19-28. [CrossRef]

17. Barth, J.; Buback, M. SP-PLP-EPR-A novel method for detailed studies into the termination kinetics of radical polymerization. Macromol. React. Eng. 2010, 4, 288-301. [CrossRef]

18. Barner-Kowollik, C.; Beuermann, S.; Buback, M.; Castignolles, P.; Charleux, B.; Coote, M.L.; Hutchinson, R.A.; Junkers, T.; Lacik, I.; Russell, G.T.; et al. Critically evaluated rate coefficients in radical polymerization-7. Secondary-radical propagation rate coefficients for methyl acrylate in the bulk. Polym. Chem. 2014, 5, 204-212. [CrossRef]

19. Beuermann, S.; Buback, M.; Hesse, P.; Kuchta, F.-D.; Lacik, I.; van Herk, A.M. Critically evaluated rate coefficients for free-radical polymerization, 6. Propagation rate coefficient of methacrylic acid in aqueous solution. Pure Appl. Chem. 2007, 79, 1463-1469. [CrossRef]

20. Buback, M.; Gilbert, R.G.; Russell, G.T.; Hill, D.J.T.; Moad, G.; Odriscoll, K.F.; Shen, J.; Winnik, M.A. Consistent values of rate parameters in free radical polymerization systems. II. Outstanding dilemmas and recommendations. J. Polym. Sci. Part A 1992, 30, 851-863. [CrossRef]

21. Beuermann, S.; Buback, M.; Davis, T.P.; Gilbert, R.G.; Hutchinson, R.A.; Olaj, O.F.; Russell, G.T.; Schweer, J.; van Herk, A.M. Critically evaluated rate coefficients for free-radical polymerization, 2. Propagation rate coefficients for methyl methacrylate. Macromol. Chem. Phys. 1997, 198, 1545-1560. [CrossRef] 
22. Beuermann, S.; Buback, M.; Davis, T.P.; Gilbert, R.G.; Hutchinson, R.A.; Kajiwara, A.; Klumperman, B.; Russell, G.T. Critically evaluated rate coefficients for free-radical polymerization, 3. Propagation rate coefficients for alkyl methacrylates. Macromol. Chem. Phys. 2000, 201, 1355-1364. [CrossRef]

23. Junkers, T.; Schneider-Baumann, M.; Koo, S.S.P.; Castignolles, P.; Barner-Kowollik, C. Determination of propagation rate coefficients for methyl and 2-ethylhexyl acrylate via high frequency PLP-SEC under consideration of the impact of chain branching. Macromolecules 2010, 43, 10427-10434. [CrossRef]

24. Mavroudakis, E.; Liang, K.; Moscatelli, D.; Hutchinson, R.A. A combined computational and experimental study on the free-radical copolymerization of styrene and hydroxyethyl acrylate. Macromol. Chem. Phys. 2012, 213, 1706-1716. [CrossRef]

25. Buback, M.; Kurz, C.H. Free-Radical propagation rate coefficients for cyclohexyl methacrylate, glycidyl methacrylate and 2-hydroxyethyl methacrylate homopolymerizations. Macromol. Chem. Phys. 1998, 199, 2301-2310. [CrossRef]

26. Hutchinson, R.A.; Beuermann, S.; Paquet, D.A.; McMinn, J.H. Determination of free-radical propagation rate coefficients for alkyl methacrylates by pulsed-laser polymerization. Macromolecules 1997, 30, 3490-3493. [CrossRef]

27. Siegmann, R.; Jelicic, A.; Beuermann, S. Propagation and termination kinetics of pegylated methacrylate radical polymerizations. Macromol. Chem. Phys. 2010, 211, 546-562. [CrossRef]

28. Beuermann, S.; Buback, M.; Davis, T.P.; García, N.; Gilbert, R.G.; Hutchinson, R.A.; Kajiwara, A.; Kamachi, M.; Lacík, I.; Russell, G.T. Critically evaluated rate coefficients for free-radical polymerization, 4. Macromol. Chem. Phys. 2003, 204, 1338-1350. [CrossRef]

29. Lacík, I.; Beuermann, S.; Buback, M. PLP-SEC study into free-radical propagation rate of nonionized acrylic acid in aqueous solution. Macromolecules 2003, 36, 9355-9363. [CrossRef]

30. Uhelska, L.; Chorvat, D.; Hutchinson, R.A.; Santanakrishnan, S.; Buback, M.; Lacik, I. Radical propagation kinetics of $N$-vinylpyrrolidone in organic solvents studied by pulsed-laser polymerization-size-exclusion chromatography (PLP-SEC). Macromol. Chem. Phys. 2014, 215, 2327-2336. [CrossRef]

31. Stach, M.; Lacik, I.; Chorvat, D.; Buback, M.; Hesse, P.; Hutchinson, R.A.; Tang, L. Propagation rate coefficient for radical polymerization of $N$-vinyl pyrrolidone in aqueous solution obtained by PLP-SEC. Macromolecules 2008, 41, 5174-5185. [CrossRef]

32. Stach, M.; Lacik, I.; Kasak, P.; Chorvat, D.; Saunders, A.J.; Santanakrishnan, S.; Hutchinson, R.A. Free-radical propagation kinetics of $N$-vinyl formamide in aqueous solution studied by PLP-SEC. Macromol. Chem. Phys. 2010, 211, 580-593. [CrossRef]

33. Seabrook, S.A.; Tonge, M.P.; Gilbert, R.G. Pulsed laser polymerization study of the propagation kinetics of acrylamide in water. J. Polym. Sci. Part A 2005, 43, 1357-1368. [CrossRef]

34. Muratore, L.M.; Coote, M.L.; Davis, T.P. Determination of the propagation rate coefficient for 3-(tris(trimethylsilyloxy)silyl) propyl methacrylate by pulsed-laser polymerization. Polymer 2000, 41, 1441-1447. [CrossRef] 
35. Yin, M.; Davis, T.P.; Heuts, J.P.A.; Barner-Kowollik, C. Propagation and termination rate coefficients in $N$-vinylcarbazole free radical polymerization obtained via $440 \mathrm{~nm}$ pulsed laser and online ${ }^{1} \mathrm{H}$ NMR kinetic experiments. Macromol. Rapid Commun. 2003, 24, 408-412. [CrossRef]

36. Balic, R.; Gilbert, R.G.; Zammit, M.D.; Davis, T.P.; Miller, C.M. Propagation rate coefficient of vinyl neo-decanoate by pulsed laser polymerization. Macromolecules 1997, 30, 3775-3780. [CrossRef]

37. Hutchinson, R.A.; Paquet, D.A.; McMinn, J.H.; Beuermann, S.; Fuller, R.E.; Jackson, C. The application of pulsed-laser methods for the determination of free-radical polymerization rate coefficients. In 5th International Workshop on Polymer Reaction Engineering; Reichert, K.H., Moritz, H.U., Eds.; John Wiley \& Sons: Hoboken, NJ, USA, 1995; Volume 131, pp. 467-492.

38. Olaj, O.F.; Schnollbitai, I. Laser-flash-initiated polymerization as a tool for evaluating (individual) kinetic constants of free-radical polymerization-5. Complete analysis by means of a single experiment. Eur. Poly. J. 1989, 25, 635-641. [CrossRef]

39. Shipp, D.A.; Smith, T.A.; Solomon, D.H.; Moad, G. Evaluation of propagation rate constants for the free radical polymerization of methacrylonitrile by pulsed laser photolysis. Macromol. Rapid Commun. 1995, 16, 837-844. [CrossRef]

40. Beuermann, S.; Paquet, D.A.; McMinn, J.H.; Hutchinson, R.A. Propagation kinetics of methacrylic acid studied by pulsed-laser polymerization. Macromolecules 1997, 30, 194-197. [CrossRef]

41. Junkers, T.; Koo, S.P.S.; Barner-Kowollik, C. Determination of the propagation rate coefficient of acrylonitrile. Polym. Chem. 2010, 1, 438-441. [CrossRef]

42. Buback, M.; Gilbert, R.G.; Hutchinson, R.A.; Klumperman, B.; Kuchta, F.-D.; Manders, B.G.; O’Driscoll, K.F.; Russell, G.T.; Schweer, J. Critically evaluated rate coefficients for free-radical polymerization, 1. Propagation rate coefficient for styrene. Macromol. Chem. Phys. 1995, 196, 3267-3280. [CrossRef]

43. Coote, M.L.; Davis, T.P. Propagation kinetics of para-substituted styrenes: A test of the applicability of the hammett relationship to free-radical polymerization. Macromolecules 1999, 32, 4290-4298. [CrossRef]

44. Hutchinson, R.A.; Aronson, M.T.; Richards, J.R. Analysis of pulsed-laser-generated molecular-weight distributions for the determination of propagation rate coefficients. Macromolecules 1993, 26, 6410-6415. [CrossRef]

45. Coote, M.L.; Lin, C.Y. Computational methods for modeling free-radical polymerization. In Computational Methods for Large Systems: Electronic Structure Approaches for Biotechnology and Nanotechnology; Reimers, J.R., Ed.; John Wiley \& Sons: Hoboken, NJ, USA, 2011; pp. 435-474.

46. Te Velde, G.; Bickelhaupt, F.M.; Baerends, E.J.; Guerra, C.F.; van Gisbergen, S.J.A.; Snijders, J.G.; Ziegler, T. Chemistry with ADF. J. Comput. Chem. 2001, 22, 931-967. [CrossRef]

47. Gordon, M.S.; Schmidt, M.W. Advances in electronic structure theory: Gamess a decade later. In Theory and Applications of Computational Chemistry: The First Forty Years; Dykstra, C.E., Frenking, G., Kim, K.S., Scuseria, G.E., Eds.; Elsevier: Amsterdam, The Netherlands, 2005; pp. 1167-1189. 
48. Frisch, M.J.; Trucks, G.W.; Schlegel, H.B.; Scuseria, G.E.; Robb, M.A.; Cheeseman, J.R.; Scalmani, G.; Barone, V.; Mennucci, B.; Petersson, G.A.; et al. Gaussian 09; Gaussian Inc.: Wallingford, CT, USA, 2009.

49. Bochevarov, A.D.; Harder, E.; Hughes, T.F.; Greenwood, J.R.; Braden, D.A.; Philipp, D.M.; Rinaldo, D.; Halls, M.D.; Zhang, J.; Friesner, R.A. Jaguar: A high-performance quantum chemistry software program with strengths in life and materials sciences. Int. J. Quantum Chem. 2013, 113, 2110-2142. [CrossRef]

50. Aquilante, F.; de Vico, L.; Ferre, N.; Ghigo, G.; Malmqvist, P.-A.; Neogrady, P.; Pedersen, T.B.; Pitonak, M.; Reiher, M.; Roos, B.O.; et al. Software news and update molcas 7: The next generation. J. Comput. Chem. 2010, 31, 224-247. [CrossRef] [PubMed]

51. Werner, H.-J.; Knowles, P.J.; Knizia, G.; Manby, F.R.; Schuetz, M. Molpro: A general-purpose quantum chemistry program package. Wiley Interdiscip. Rev. Comput. Mol. Sci. 2012, 2, 242-253. [CrossRef]

52. Shao, Y.; Gan, Z.; Epifanovsky, E.; Gilbert, A.T.B.; Wormit, M.; Kussmann, J.; Lange, A.W.; Behn, A.; Deng, J.; Feng, X.; et al. Advances in molecular quantum chemistry contained in the Q-Chem 4 program package. Mol. Phys. 2015, 113, 184-215. [CrossRef]

53. Agarwal, M.; Frank, M.I. SPARTAN: A software tool for parallelization bottleneck analysis. In Proceedings of the 2009 ICSE Workshop on Multicore Software Engineering, Vancouver, BC, Canada, 18 May 2009; pp. 56-63.

54. Furche, F.; Ahlrichs, R.; Haettig, C.; Klopper, W.; Sierka, M.; Weigend, F. Turbomole. Wiley Interdiscip. Rev. Comput. Mol. Sci. 2014, 4, 91-100. [CrossRef]

55. Deumens, E.; Lotrich, V.F.; Perera, A.; Ponton, M.J.; Sanders, B.A.; Bartlett, R.J. Software design of aces III with the super instruction architecture. Wiley Interdiscip. Rev.Comput. Mol. Sci. 2011, 1, 895-901. [CrossRef]

56. Mohr, S.; Ratcliff, L.E.; Boulanger, P.; Genovese, L.; Caliste, D.; Deutsch, T.; Goedecker, S. Daubechies wavelets for linear scaling density functional theory. J. Chem. Phys. 2014, 140, 204110. [CrossRef] [PubMed]

57. Genovese, L.; Neelov, A.; Goedecker, S.; Deutsch, T.; Ghasemi, S.A.; Willand, A.; Caliste, D.; Zilberberg, O.; Rayson, M.; Bergman, A.; et al. Daubechies wavelets as a basis set for density functional pseudopotential calculations. J. Chem. Phys. 2008, 129, 014109. [CrossRef] [PubMed]

58. Hutter, J.; Iannuzzi, M.; Schiffmann, F.; VandeVondele, J. CP2K: Atomistic simulations of condensed matter systems. Wiley Interdiscip. Rev. Comput. Mol. Sci. 2014, 4, 15-25. [CrossRef]

59. Matsen, F.A. Spin-free quantum-chemistry. XXIV. Fermion Many-Body Theory. Int. J. Quantum Chem. 1987, 32, 87-104. [CrossRef]

60. Fock, V. Approximation method for the solution of the quantum mechanical multibody problems. Z. Fur Phys. 1930, 61, 126-148. [CrossRef]

61. Hohenberg, P.; Kohn, W. Inhomogeneous electron gas. Phys. Rev. B 1964, 136, B864. [CrossRef]

62. Fischer, H.; Radom, L. Factors controlling the addition of carbon-centered radicals to alkenes-An experimental and theoretical perspective. Angew. Chem. Int. Edit. 2001, 40, 1340-1371. [CrossRef] 
63. Toh, J.S.S.; Huang, D.M.; Lovell, P.A.; Gilbert, R.G. Ab initio calculation of the rate coefficient for short-chain branching in free-radical polymerizations. Polymer 2001, 42, 1915-1920. [CrossRef]

64. Gomez-Balderas, R.; Coote, M.L.; Henry, D.J.; Radom, L. Reliable theoretical procedures for calculating the rate of methyl radical addition to carbon-carbon double and triple bonds. J. Phys. Chem. A 2004, 108, 2874-2883. [CrossRef]

65. Heuts, J.P.A.; Gilbert, R.G.; Radom, L. Determination of arrhenius parameters for propagation in free-radical polymerizations: An assessment of ab initio procedures. J. Phys. Chem. 1996, 100, 18997-19006. [CrossRef]

66. Dossi, M.; Storti, G.; Moscatelli, D. Initiation kinetics in free-radical polymerization: Prediction of thermodynamic and kinetic parameters based on ab initio calculations. Macromol. Theory Simul. 2010, 19, 170-178. [CrossRef]

67. Fascella, S.; Cavallotti, C.; Rota, R.; Carra, S. Quantum chemistry investigation of key reactions involved in the formation of naphthalene and indene. J. Phys. Chem. A 2004, 108, 3829-3843. [CrossRef]

68. Van Speybroeck, V.; van Neck, D.; Waroquier, M.; Wauters, S.; Saeys, M.; Marin, G.B. Ab initio study of radical addition reactions: Addition of a primary ethylbenzene radical to ethene (I). J. Phys. Chem. A 2000, 104, 10939-10950. [CrossRef]

69. Peng, C.Y.; Ayala, P.Y.; Schlegel, H.B.; Frisch, M.J. Using redundant internal coordinates to optimize equilibrium geometries and transition states. J. Comput. Chem. 1996, 17, 49-56. [CrossRef]

70. Cuccato, D.; Mavroudakis, E.; Moscatelli, D. Quantum chemistry investigation of secondary reaction kinetics in acrylate-based copolymers. J. Phys. Chem. A 2013, 117, 4358-4366. [CrossRef] [PubMed]

71. Coote, M.L. Quantum-chemical modeling of free-radical polymerization. Macromol. Theory Simul. 2009, 18, 388-400. [CrossRef]

72. Noble, B.B.; Coote, M.L. First principles modelling of free-radical polymerisation kinetics. Int. Rev. Phys. Chem. 2013, 32, 467-513. [CrossRef]

73. Fueno, T.; Kamachi, M. Ab initio SCF study of addition of the methyl radical to vinyl compounds. Macromolecules 1988, 21, 908-912. [CrossRef]

74. Gonzalez, C.; Sosa, C.; Schlegel, H.B. Ab initio Study of the addition-reaction of the methyl radical to the ethylene and formaldehyde. J. Phys. Chem. 1989, 93, 2435-2440. [CrossRef]

75. Arnaud, R.; Subra, R.; Barone, V.; Lelj, F.; Olivella, S.; Sole, A.; Russo, N. Ab-initio mechanistic studies of radical reactions. Directive effects in the addition of methyl radical to unsymmetrical fluoroathenes. J. Chem. Soc. Perkin Trans. 2 1986. [CrossRef]

76. Wong, M.W.; Pross, A.; Radom, L. Comparison of the addition of $\mathrm{CH}_{3}$.bul., $\mathrm{CH}_{2} \mathrm{OH}$.bul., and $\mathrm{CH}_{2}$ CN.bul. Radicals to substituted alkenes: A theoretical study of the reaction mechanism. J. Am. Chem. Soc. 1994, 116, 6284-6292. [CrossRef]

77. Heuts, J.P.A.; Gilbert, R.G.; Radom, L. A Priori prediction of propagation rate coefficients in free-radical polymerizations: Propagation of ethylene. Macromolecules 1995, 28, 8771-8781. [CrossRef] 
78. Wong, M.W.; Radom, L. Radical addition to alkenes: Further assessment of theoretical procedures. J. Phys. Chem. A 1998, 102, 2237-2245. [CrossRef]

79. Coote, M.L.; Davis, T.P.; Radom, L. Conformational dependence of the penultimate unit effect in free-radical copolymerization. Macromolecules 1999, 32, 5270-5276. [CrossRef]

80. Coote, M.L.; Davis, T.P.; Radom, L. Effect of the penultimate unit on radical stability and reactivity in free-radical polymerization. Macromolecules 1999, 32, 2935-2940. [CrossRef]

81. Coote, M.L.; Davis, T.P.; Radom, L. The Effect of remote substituents in free radical addition reactions: New evidence for the penultimate unit effect. J. Mol. Struct. 1999, 461, 91-96. [CrossRef]

82. Moscatelli, D.; Dossi, M.; Cavallotti, C.; Storti, G. Density functional theory study of addition reactions of carbon-centered radicals to alkenes. J. Phys. Chem. A 2011, 115, 52-62. [CrossRef] [PubMed]

83. Hirunsit, P.; Balbuena, P.B. Addition reactions of alkyl and carboxyl radicals to vinylidene fluoride. J. Phys. Chem. A 2008, 112, 4483-4489. [CrossRef] [PubMed]

84. Van Speybroeck, V.; van Cauter, K.; Coussens, B.; Waroquier, M. Ab initio study of free-radical polymerizations: Cost-effective methods to determine the reaction rates. ChemPhysChem 2005, 6, 180-189. [CrossRef] [PubMed]

85. Van Cauter, K.; van Speybroeck, V.; Vansteenkiste, P.; Reyniers, M.F.; Waroquier, M. Ab initio study of free-radical polymerization: Polyethylene propagation kinetics. ChemPhysChem 2006, 7, 131-140. [CrossRef] [PubMed]

86. Izgorodina, E.I.; Coote, M.L. Accurate $a b$ initio prediction of propagation rate coefficients in free-radical polymerization: Acrylonitrile and vinyl chloride. Chem. Phys. 2006, 324, 96-110. [CrossRef]

87. Van Cauter, K.; van Speybroeck, V.; Waroquier, M. Ab initio study of poly(vinyl chloride) propagation kinetics: Head-to-head versus head-to-tail additions. ChemPhysChem 2007, 8, 541-552. [CrossRef] [PubMed]

88. Huang, D.M.; Monteiro, M.J.; Gilbert, R.G. A theoretical study of propagation rate coefficients for methacrylonitrile and acrylonitrile. Macromolecules 1998, 31, 5175-5187. [CrossRef]

89. Yu, X.R.; Pfaendtner, J.; Broadbelt, L.J. Ab initio study of acrylate polymerization reactions: Methyl methacrylate and methyl acrylate propagation. J. Phys. Chem. A 2008, 112, 6772-6782. [CrossRef] [PubMed]

90. Gunaydin, H.; Salman, S.; Tuzun, N.S.; Avci, D.; Aviyente, V. Modeling the free radical polymerization of acrylates. Int. J. Quantum Chem. 2005, 103, 176-189. [CrossRef]

91. Degirmenci, I.; Aviyente, V.; van Speybroeck, V.; Waroquier, M. DFT study on the propagation kinetics of free-radical polymerization of $\alpha$-substituted acrylates. Macromolecules 2009, 42, 3033-3041. [CrossRef]

92. Isik, M.; Karahan, O.; Avci, D.; Aviyente, V. A computational approach to the free radical polymerization kinetics of alkyl $\alpha$-hydroxymethacrylate monomers: A structure-reacti vity relationship. J. Polym. Sci. Part A 2013, 51, 2375-2384. [CrossRef] 
93. Dogan, B.; Catak, S.; van Speybroeck, V.; Waroquier, M.; Aviyente, V. Free radical polymerization of ethyl methacrylate and ethyl $\alpha$-hydroxy methacrylate: A computational approach to the propagation kinetics. Polymer 2012, 53, 3211-3219. [CrossRef]

94. Karahan, O.; Avci, D.; Aviyente, V. Structure-reactivity relationships of alkyl $\alpha$-hydroxymethacrylate derivatives. J. Polym. Sci. Part A 2011, 49, 3058-3068. [CrossRef]

95. Moscatelli, D.; Dossi, M.; Cavallotti, C.; Storti, G. Ab initio calculation of the propagation kinetics in free radical polymerization: Chain length and penultimate effects. Macromol. Symp. 2007, 259, 337-347. [CrossRef]

96. Dossi, M.; Storti, G.; Moscatelli, D. Quantum chemistry: A powerful tool in polymer reaction engineering. Macromol. Symp. 2011, 302, 16-25. [CrossRef]

97. Deglmann, P.; Muller, I.; Becker, F.; Schafer, A.; Hungenberg, K.D.; Weiss, H. Prediction of propagation rate coefficients in free radical solution polymerization based on accurate quantum chemical methods: Vinylic and related monomers, including acrylates and acrylic acid. Macromol. React. Eng. 2009, 3, 496-515. [CrossRef]

98. Liang, K.; Dossi, M.; Moscatelli, D.; Hutchinson, R.A. An investigation of free-radical copolymerization propagation kinetics of styrene and 2-hydroxyethyl methacrylate. Macromolecules 2009, 42, 7736-7744. [CrossRef]

99. Lin, C.Y.; Izgorodina, E.I.; Coote, M.L. First principles prediction of the propagation rate coefficients of acrylic and vinyl esters: Are we there yet? Macromolecules 2010, 43, 553-560. [CrossRef]

100. Rooney, T.R.; Mavroudakis, E.; Lacik, I.; Hutchinson, R.A.; Moscatelli, D. Pulsed-laser and quantum mechanics study of $N$-butyl cyanoacrylate and methyl methacrylate free-radical copolymerization. Polym. Chem. 2015, 6, 1594-1603. [CrossRef]

101. Dossi, M.; Liang, K.; Hutchinson, R.A.; Moscatelli, D. Investigation of free-radical copolymerization propagation kinetics of vinyl acetate and methyl methacrylate. J. Phys. Chem. B 2010, 114, 4213-4222. [CrossRef] [PubMed]

102. Mavroudakis, E.; Cuccato, D.; Dossi, M.; Comino, G.; Moscatelli, D. Quantum chemistry investigation of fluorinated polymer systems of industrial interest. J. Phys. Chem. A 2014, 118, 238-247. [CrossRef] [PubMed]

103. Thickett, S.C.; Gilbert, R.G. Propagation rate coefficient of acrylic acid: Theoretical investigation of the solvent effect. Polymer 2004, 45, 6993-6999. [CrossRef]

104. Cuccato, D.; Dossi, M.; Moscatelli, D.; Storti, G. Quantum chemical investigation of secondary reactions in poly(vinyl chloride) free-radical polymerization. Macromol. React. Eng. 2012, 6, 330-345. [CrossRef]

105. Cuccato, D.; Dossi, M.; Moscatelli, D.; Storti, G. A density functional theory study of poly(vinyl chloride) (PVC) free radical polymerization. Macromol. Symp. 2011, 302, 100-109. [CrossRef]

106. Van Cauter, K.; van den Bossche, B.J.; van Speybroeck, V.; Waroquier, M. Ab initio study of free-radical polymerization: Defect structures in poly(vinyl chloride). Macromolecules 2007, 40, 1321-1331. [CrossRef] 
107. Filley, J.; McKinnon, J.T.; Wu, D.T.; Ko, G.H. Theoretical study of ethylene-vinyl acetate free-radical copolymerization: reactivity ratios, penultimate effects, and relative rates of chain transfer to polymer. Macromolecules 2002, 35, 3731-3738. [CrossRef]

108. Mayo, F.R.; Lewis, F.M. Copolymerization. I. A basis for comparing the behavior of monomers in copolymerization; the copolymerization of styrene and methyl methacrylate. J. Am. Chem. Soc. 1944, 66, 1594-1601. [CrossRef]

109. Merz, E.; Alfrey, T.; Goldfinger, G. Intramolecular reactions in vinyl polymers as a means of investigation of the propagation step. J. Polym. Sci. 1946, 1, 75-82. [CrossRef]

110. Fukuda, T.; Ma, Y.D.; Inagaki, H. Free-radical copolymerization. 3. Determination of rate constants of propagation and termination for styrene/methyl methacrylate system. A critical test of terminal-model kinetics. Macromolecules 1985, 18, 17-26. [CrossRef]

111. Mitoraj, M.; Michalak, A. DFT studies on isomerization reactions in the copolymerization of ethylene and methyl acrylate catalyzed by Ni-diimine and Pd-diimine complexes. J. Mol. Model. 2005, 11, 341-350. [CrossRef] [PubMed]

112. Wang, W.; Tanaka, T.; Tsubota, M.; Fujiki, M.; Yamanaka, S.; Nomura, K. Effect of cyclopentadienyl fragment in copolymerization of ethylene with cyclic olefins catalyzed by non-bridged (aryloxo)(cyclopentadienyl)titanium(IV) complexes. Adv. Synth. Catal. 2005, 347, 433-446. [CrossRef]

113. Bebe, S.; Yu, X.R.; Hutchinson, R.A.; Broadbelt, L.J. Estimation of free radical polymerization rate coefficients using computational chemistry. Macromol. Symp. 2006, 243, 179-189. [CrossRef]

114. Dossi, M.; Moscatelli, D. A QM approach to the calculation of reactivity ratios in free-radical copolymerization. Macromol. React. Eng. 2012, 6, 74-84. [CrossRef]

115. Yu, X.; Levine, S.E.; Broadbelt, L.J. Kinetic study of the copolymerization of methyl methacrylate and methyl acrylate using quantum chemistry. Macromolecules 2008, 41, 8242-8251. [CrossRef]

116. Coote, M.L.; Johnston, L.P.M.; Davis, T.P. Copolymerization propagation kinetics of styrene and methyl methacrylate-revisited. 2. Kinetic analysis. Macromolecules 1997, 30, 8191-8204. [CrossRef]

117. Furuncuoglu Ozaltin, T.; Dereli, B.; Karahan, O.; Salman, S.; Aviyente, V. Solvent effects on free-radical copolymerization of styrene and 2-hydroxyethyl methacrylate: A DFT study. New J. Chem. 2014, 38, 170-178. [CrossRef]

118. Wang, W.; Hutchinson, R.A. PLP/SEC/NMR study of free radical copolymerization of styrene and glycidyl methacrylate. Macromolecules 2008, 41, 9011-9018. [CrossRef]

119. Li, D.; Li, N.; Hutchinson, R.A. High-temperature free radical copolymerization of styrene and butyl methacrylate with depropagation and penultimate kinetic effects. Macromolecules 2006, 39, 4366-4373. [CrossRef]

120. Dossi, M.; Storti, G.; Moscatelli, D. A quantum chemistry study of the free-radical copolymerization propagation kinetics of styrene and 2-hydroxyethyl acrylate. Polym. Eng. Sci. 2011, 51, 2109-2114. [CrossRef] 
121. McManus, N.T.; Kim, J.D.; Penlidis, A. Observations on styrene-hydroxyethyl acrylate and styrene-hydroxyethyl acrylate-ethyl acrylate polymerizations. Polym. Bull. 1998, 41, 661-668. [CrossRef]

122. Davis, T.P.; O'Driscoll, K.F.; Piton, M.C.; Winnik, M.A. Copolymerization propagation kinetics of styrene with alkyl acrylates. Polym. Int. 1991, 24, 65-70. [CrossRef]

123. Zubov, V.P.; Valuev, L.I.; Kabanov, V.A.; Kargin, V.A. Effects of complexing agents in radical copolymerization. J. Polym. Sci. Part A 1971, 9, 833-854. [CrossRef]

124. Jenkins, A.D.; Tsartolia, E.; Walton, D.R.M.; Stejskal, J.; Kratochvil, P. Statistical group-transfer copolymerization. 1. Monomer reactivity ratios for normal-butyl methacrylate and methyl-methacrylate. Polym. Bull. 1988, 20, 97-100.

125. Brar, A.S.; Charan, S. Sequence determination of vinyl-acetate methyl acrylate copolymers by NMR-spectroscopy. J. Appl. Polym. Sci. 1994, 53, 1813-1822. [CrossRef]

126. Ma, Y.D.; Won, Y.C.; Kubo, K.; Fukuda, T. Propagation and termination processes in the free-radical copolymerization of methyl methacrylate and vinyl acetate. Macromolecules 1993, 26, 6766-6770. [CrossRef]

127. Rätzsch, M.; Schneider, W.; Musche, D. Reactivity of ethylene in the radically initiated copolymerization of ethylene with vinylacetate. J. Polym. Sci. Part A 1971, 9, 785-790. [CrossRef]

128. Gelin, M.P.; Ameduri, B. Radical solution copolymerisation of vinylidene fluoride with hexafluoropropene. J. Fluor. Chem. 2005, 126, 577-585. [CrossRef]

129. Moggi, G.; Bonardelli, P.; Bart, J.C.J. Copolymers of 1,1-difluoroethene with tetrafluoroethene, chlorotrifluoroethene, and bromotrifluoroethene. J. Polym. Sci. Part B 1984, 22, 357-365. [CrossRef]

130. Logothetis, A.L. Chemistry of fluorocarbon elastomers. Prog. Polym. Sci. 1989, 14, 251-296. [CrossRef]

131. De Sterck, B.; Vaneerdeweg, R.; Du Prez, F.; Waroquier, M.; van Speybroeck, V. Solvent effects on free radical polymerization reactions: The influence of water on the propagation rate of acrylamide and methacrylamide. Macromolecules 2010, 43, 827-836. [CrossRef]

132. Degirmenci, I.; Ozaltin, T.F.; Karahan, O.; van Speybroeck, V.; Waroquier, M.; Aviyente, V. Origins of the solvent effect on the propagation kinetics of acrylic acid and methacrylic Acid. $J$. Polym. Sci. Part A 2013, 51, 2024-2034. [CrossRef]

133. Degirmenci, I.; Eren, S.; Aviyente, V.; de Sterck, B.; Hemelsoet, K.; van Speybroeck, V.; Waroquier, M. Modeling the solvent effect on the tacticity in the free radical polymerization of methyl methacrylate. Macromolecules 2010, 43, 5602-5610. [CrossRef]

134. Klamt, A.; Schuurmann, G. Cosmo: A new approach to dielectric screening in solvents with explicit expressions for the screening energy and its gradient. J. Chem. Soc. Perkin Trans. 2 1993. [CrossRef]

135. Miertuš, S.; Scrocco, E.; Tomasi, J. Electrostatic interaction of a solute with a continuum. A direct utilizaion of ab initio molecular potentials for the prevision of solvent effects. Chem. Phys. 1981, 55, 117-129. [CrossRef] 
136. Ensing, B.; de Vivo, M.; Liu, Z.; Moore, P.; Klein, M.L. Metadynamics as a tool for exploring free energy landscapes of chemical reactions. Acc. Chem. Res. 2006, 39, 73-81. [CrossRef] [PubMed]

137. Degirmenci, I.; Avci, D.; Aviyente, V.; van Cauter, K.; van Speybroeck, V.; Waroquier, M. Density functional theory study of free-radical polymerization of acrylates and methacrylates: Structure-reactivity relationship. Macromolecules 2007, 40, 9590-9602. [CrossRef]

138. Hemelsoet, K.; Moran, D.; van Speybroeck, V.; Waroquier, M.; Radom, L. An assessment of theoretical procedures for predicting the thermochemistry and kinetics of hydrogen abstraction by methyl radical from benzene. J. Phys. Chem. A 2006, 110, 8942-8951. [CrossRef] [PubMed]

139. Saeys, M.; Reyniers, M.F.; van Speybroeck, V.; Waroquier, M.; Marin, G.B. Ab initio group contribution method for activation energies of hydrogen abstraction reactions. ChemPhysChem 2006, 7, 188-199. [CrossRef] [PubMed]

140. Moghadam, N.; Liu, S.; Srinivasan, S.; Grady, M.C.; Soroush, M.; Rappe, A.M. Computational study of chain transfer to monomer reactions in high-temperature polymerization of alkyl acrylates. J. Phys. Chem. A 2013, 117, 2605-2618. [CrossRef] [PubMed]

141. Purmova, J.; Pauwels, K.F.D.; Agostini, M.; Bruinsma, M.; Vorenkamp, E.J.; Schouten, A.J.; Coote, M.L. Experimental and theoretical evaluation of the reactions leading to formation of internal double bonds in suspension PVC. Macromolecules 2008, 41, 5527-5539. [CrossRef]

142. Pfaendtner, J.; Yu, X.R.; Broadbelt, L.J. Quantum chemical investigation of low-temperature intramolecular hydrogen transfer reactions of hydrocarbons. J. Phys. Chem. A 2006, 110, 10863-10871. [CrossRef] [PubMed]

143. Liu, S.; Srinivasan, S.; Grady, M.C.; Soroush, M.; Rappe, A.M. Backbiting and $\beta$-scission reactions in free-radical polymerization of methyl acrylate. Int. J. Quantum Chem. 2014, 114, 345-360. [CrossRef]

144. Cuccato, D.; Mavroudakis, E.; Dossi, M.; Moscatelli, D. A density functional theory study of secondary reactions in $N$-butyl acrylate free radical polymerization. Macromol. Theory Simul. 2013, 22, 127-135. [CrossRef]

145. Yu, X.R.; Broadbelt, L.J. Kinetic study of 1,5-hydrogen transfer reactions of methyl acrylate and butyl acrylate using quantum chemistry. Macromol. Theory Simul. 2012, 21, 461-469. [CrossRef]

146. Karahan, O.; Isik, M.; Cifci, G.; Ugur, I.; Avci, D.; Aviyente, V. Cyclization tendencies in the free radical polymerization of allyl acrylate derivatives: A computational study. J. Polym. Sci. Part A 2011, 49, 2474-2483. [CrossRef]

147. Moghadam, N.; Srinivasan, S.; Grady, M.C.; Rappe, A.M.; Soroush, M. Theoretical study of chain transfer to solvent reactions of alkyl acrylates. J. Phys. Chem. A 2014, 118, 5474-5487. [CrossRef] [PubMed]

148. Furuncuoglu, T.; Ugur, I.; Degirmenci, I.; Aviyente, V. Role of chain transfer agents in free radical polymerization kinetics. Macromolecules 2010, 43, 1823-1835. [CrossRef]

149. Mavroudakis, E.; Cuccato, D.; Moscatelli, D. Theoretical study of chain transfer to agent kinetics in butyl acrylate polymerization. Ind. Eng. Chem. Res. 2014, 53, 9058-9066. [CrossRef] 
150. Mavroudakis, E.; Cuccato, D.; Moscatelli, D. Quantum mechanical investigation on bimolecular hydrogen abstractions in butyl acrylate-based free radical polymerization processes. J. Phys. Chem. A 2014, 118, 1799-1806. [CrossRef] [PubMed]

151. Moscatelli, D.; Cavallotti, C.; Morbidelli, M. Prediction of molecular weight distributions based on ab initio calculations: Application to the high temperature styrene polymerization. Macromolecules 2006, 39, 9641-9653. [CrossRef]

152. Dossi, M.; Storti, G.; Moscatelli, D. Relevance of backbiting and $\beta$-scission reactions in the free radical polymerization of acrylonitrile. Macromol. Symp. 2010, 289, 119-123. [CrossRef]

153. Liu, S.; Srinivasan, S.; Grady, M.C.; Soroush, M.; Rappe, A.M. Computational study of cyclohexanone-monomer co-initiation mechanism in thermal homo-polymerization of methyl acrylate and methyl methacrylate. J. Phys. Chem. A 2012, 116, 5337-5348. [CrossRef] [PubMed]

154. Srinivasan, S.; Lee, M.W.; Grady, M.C.; Soroush, M.; Rappe, A.M. Computational evidence for self-initiation in spontaneous high-temperature polymerization of methyl methacrylate. J. Phys. Chem. A 2011, 115, 1125-1132. [CrossRef] [PubMed]

155. Cuccato, D.; Dossi, M.; Polino, D.; Cavallotti, C.; Moscatelli, D. Is quantum tunneling relevant in free-radical polymerization? Macromol. React. Eng. 2012, 6, 496-506. [CrossRef]

156. Eckart, C. The penetration of a potential barrier by electrons. Phys. Rev. 1930, 35, 1303-1309. [CrossRef]

157. Maeder, S.; Gilbert, R.G. Measurement of transfer constant for butyl acrylate free-radical polymerization. Macromolecules 1998, 31, 4410-4418. [CrossRef]

(C) 2015 by the authors; licensee MDPI, Basel, Switzerland. This article is an open access article distributed under the terms and conditions of the Creative Commons Attribution license (http://creativecommons.org/licenses/by/4.0/). 